This item was submitted to Loughborough's Research Repository by the author.

Items in Figshare are protected by copyright, with all rights reserved, unless otherwise indicated.

\title{
Cell-signalling repression in bacterial quorum sensing
}

PLEASE CITE THE PUBLISHED VERSION

\section{LICENCE}

CC BY-NC-ND 4.0

\section{REPOSITORY RECORD}

Ward, John P., J.R. King, A.J. Koerber, J.M. Croft, R.E. Sockett, and P. Williams. 2019. "Cell-signalling Repression in Bacterial Quorum Sensing". figshare. https://hdl.handle.net/2134/314. 


\title{
Cell-signalling repression in bacterial quorum sensing
}

\author{
J.P. Ward, \\ Mathematical Biology Group, Department of Mathematical Sciences, Loughborough University, \\ Loughborough, LE11 3TU, UK. \\ J.R. King, A.J. Koerber, \\ Division of Theoretical Mechanics, School of Mathematical Sciences, \\ University of Nottingham, Nottingham, NG7 2RD, UK. \\ J.M. Croft, R.E. Sockett, \\ Division of Genetics, School of Clinical Laboratory Sciences, Queens Medical Centre, \\ University of Nottingham, Nottingham, NG7 2UH, UK. \\ and \\ P. Williams, \\ School of Pharmaceutical Sciences, University of Nottingham, Nottingham, NG7 2RD, UK.
}

\begin{abstract}
In this paper we expand on two mathematical models for investigating the role of three distinct repression mechanisms within the so called quorum sensing (QS) cell-signalling process of bacterial colonies growing (1) in liquid cultures and (2) in biofilms. The repression mechanisms studied are (i) reduction of cell signalling molecule (QSM) production by a constitutively produced agent degrading the messenger RNA of a crucial enzyme (QSE), (ii) lower QSM production rate due to a negative feedback process and (iii) loss of QSMs by binding directly to a constitutively produced agent; the first two mechanisms are known to be employed by the pathogenic bacterium Pseudomonas aeruginosa and the last is relevant to the plant pathogen Agrobacterium tumefaciens. The modelling approach assumes that the bacterial colony consists of two sub-populations, namely down- and up-regulated cells, that differ in the rates at which they produce QSMs, while QSM concentration governs the switching between sub-populations. Parameter estimates are obtained by curve-fitting experimental data (involving $P$. aeruginosa growth in liquid culture, obtained as part of this study) to solutions of model (1). Asymptotic analysis of the model (1) shows that mechanism (i) is necessary, but not sufficient, to predict the observed saturation of QSM levels in an exponentially growing colony; either mechanism (ii) or (iii) also needs to be incorporated to obtain saturation. Consequently, only a fraction of the population will become up-regulated. Furthermore, only mechanisms (i) and (iii) effect the main timescales for up regulation. Repression was found to play less significant role in a biofilms, but mechanisms (i)-(iii) were nevertheless found to reduce the ulitimate up-regulated cell fraction and mechanisms (i) and (iii) increase the timescale for substantial up regulation and decrease the wave speed of an expanding front of QS activity.
\end{abstract}

Keywords: bacteria; quorum sensing; repression; mathematical modelling; parameter estimation.

${ }^{*}$ Author to whom correspondence should be addressed. Email: john.ward@lboro.ac.uk 


\section{Introduction}

The phenomenon of quorum sensing (QS), a sophisticated cell-to-cell signalling system employed by many bacterial species, is emerging as one of the most important issues in the study of bacterial behavioural dynamics [19]. The phenomenon manifests itself in the apparent change of the behaviour (phenotype) of an entire population when the bacterial density has reached a threshold level. Such density dependent behavioural changes include the "switching on" of bioluminescence of Vibrio fischeri (a species that resides in certain deep sea squid, [11]), conjugal transfer in Agrobacterium tumefaciens (a form of sexual reproduction, [22]), swarming behaviour in Serratia liquefacians (presumably as a means of population expansion) and production of virulence factors in Burkholderia cepacia and Pseudomonas aeruginosa (both with significant medical implications for sufferers of cystic fibrosis [10] and, in the latter case, for patients with severe open wounds or burn injuries [14]). In some cases, rather than QS "switching on" a given phenotype, it may instead function by de-repressing a phenotype which is actively repressed at low densities $[2,17]$. The advantage of QS for the bacteria is that by restricting certain behavioural traits it reduces expenditure of vital nutrient resources on activities that will have no impact when the population density is small. In the case of the infection of wounds by P. aeruginosa, it is believed that QS enables the initially benign colonising bacteria to build up numbers whilst being (relatively) overlooked by the immune system; when the population finally becomes virulent, their greater numbers thus increase the chances of the immune system being overwhelmed, leading to a serious infection.

In its "simplest" form, QS is governed by two genes, one encoding an enzyme (QSE) that promotes the production of a small, freely diffusible quorum sensing molecule (QSM) and the other a cognate quorum sensing protein (QSP) [24]. A QSM can combine with a QSP to form a QSM-QSP complex which, by interacting with the promoter region of the gene (called the lux-box), enhances production of the QSM, and perhaps of the QSP, and up regulates the production of proteins that are involved in the appropriate shift in behavioural traits (for example, production of luciferase in $V$. fischeri to produce bioluminescence). Consequently, as the bacterial density increases, more QSM will be present, leading to greater up regulation of the appropriate genes within the population. That is to say, QS is a partially autoinductive process involving positive feedback loops; by "partially" we mean here that the production of QS products is limited by the number of bacteria and availability of raw materials. Figure 1 is a simple schematic of the LasR/LasI (primary) QS system of $P$. aeruginosa based on Withers et al. [27]; here the protein LasR is a QSP and 3-oxo-C12-HSL ( $N$-[3-oxododecanoyl]L-homoserine lactone) is a QSM. Such autoinductive systems could bring about the needless over-production of QSMs, QSPs and other up regulated products, leading to waste in nutrient resources. Discoveries in recent years have shown that a few species have evolved means of bypassing this problem, by repressing certain components of the QS process. The most well studied examples are those of $P$. aeruginosa $[6,7,8,21]$ and A.tumefaciens $[4,23]$. The repression mechanisms investigated in this paper are summarised below, the first two being known to be relevant for $P$. aeruginosa and the last to A. tumefaciens.

1. "Background" Inhibition (BI) of QSM output by constitutively produced regulator proteins which interfere in some way with the production or action of the QSE (which catalyses the reaction of the QSM precursors). Two such proteins have been identified in P. aeruginosa, namely QscR [6] and RsmA [21], which are produced independently 
of QS; see Figure 1. RsmA is believed to destroy messenger-RNA transcripts for many proteins [13], including that for the enzyme LasI, the QSE for the QSM 3-oxo-C12-HSL in P. aeruginosa (see Figure 2); how QscR inhibit QSM output is currently unknown.

2. Negative feedback (NF) process, whereby a protein positively regulated by QS represses QSM production. In P. aeruginosa the production of repressor protein RsaL is enhanced by QS; this protein probably binds directly to the lasI promoter, preventing expression of the gene [7] (see Figure 1).

3. "Soaking up" (SU) of QSMs by forming inert dimers with certain constitutively produced proteins. The protein TrlR in A. tumefaciens is structural similar to the bacterium's QSP (namely TraR) and can bind with a QSM to form a stable dimer [4], rendering the QSM unavailable for QS. In fact, the role of TrlR seems to be considerably more complex, as it can also bind with and thus deactivate QSPs; however, only the action of the soaking up of QSMs will be considered in this paper.

The abbreviations BI, NF and SU for the three repression mechanism will be used throughout the paper. A. tumefaciens produces at least one other molecule involved in the repression of QS, namely TraM [23], which also deactivates the QSP molecule. Curiously, QS seems to promote the production of both TraR and TraM. This apparent conflict between promotion and repression suggests that there are other, as yet undiscovered, regulators involved, perhaps processes that somehow delay the production of TraM. Since the details of how TraR, TraM and TrlR are regulated and interact is open to speculation, the process of QSP repression will not be considered in this paper.

As is now widely recognised, at the intracellular level many biological phenomena are governed by complex interactions between positive and negative feedback processes. Here we consider in detail a relatively well-characterised example which has significant medical (in particular) implications. Specifically, we consider cell signalling processes in a population of bacteria whereby positive feedback (quorum sensing) leads to concerted action by the entire colony, but negative feedback (repression) is needed if this concerted action is not to lead to excessive use of available resources. By operating in tandem, the two processes allow the colony to operate in an efficient multi-cellular fashion.

The QS process has been the subject of a number mathematical models, the approaches ranging competition models [3], through mass-action-based kinetic models of the biochemistry $[5,9,15,18]$ to macro-scale population models $[16,25,26]$. The mass action model of Dockery and Keener [9], modelling QS in P. aeruginosa is (more-or-less) as depicted in Figure 1, considered, inter alia, (a) the decay of the mRNA-lasI protein required to generate QSMs, reflecting the BI mechanism, (b) an inhibitor of mRNA-lasI, reflecting NF, and (c) the constant decay of QSMs, reflecting SU; we note, however, that there is no biological evidence that $P$. aeruginosa produces a molecule that soaks-up or cause decay of QSMs, so it is uncertain whether the SU process is relevant for this bacterium. The role of these QS repression mechanisms was not investigated systematically in [9], indeed, the negative feedback loop was neglected in order to simplify the model for further analysis; consequently no investigation into the role of feedback was attempted. The approach to the modelling in the current paper studies QS on a macroscopic level, extending the model of Ward et al. [26]. The modelling assumes that the bacteria are in one of two states, namely down-regulated and up-regulated, with QSM levels controlling the rates of conversion from one form to the other. The addition 
of the repression mechanisms involves relatively simple extensions to the original model and significantly improves qualitative (and quantitative) agreement with experimental data; thus emphasising the biological significance of these mechanisms. The model is intended to be generic and henceforth we will refer to the signalling molecules as QSMs, the QSM for $P$. aeruginosa being 3-oxo-C12-HSL.

In the next section we briefly present the QS model, describing the extensions to model the QSM repression processes. Using curve fitting techniques, we present in Section 2.2 estimates for the parameter values using experimental data from an exponentially growing bacterial colony in liquid culture. The model is non-dimensionalised and studied using asymptotic analysis based on a limit motivated by the parameter estimates. The approach to the analysis is similar to that described in Ward et al. [26] and draws out the important timescales for the evolution of system. A simple model for quorum sensing drug targeting is also studied. In Section 3 we extend the modelling described in Ward et al. [25] to investigate the role of QSM repression in early biofilm development. Again, asymptotic analysis is used to determine the effects of the repression mechanisms on the timescales for up regulation and on the travelling wave solutions discussed in [25].

\section{Role of QSM during during exponential growth}

\subsection{Mathematical model}

In this section we a propose and study a simple extension to the model of Ward et al. [26] which accounts for the three QSM repression mechanisms listed above. Many of the modelling details are discussed in [26] and will be described only briefly here. The population of bacteria is assumed to consist of two sub-populations, namely down-regulated (with density $n_{d}$ ) and upregulated (with density $n_{u}$ ) cells (so the total population $n_{T}=n_{d}+n_{u}$ ). These sub-populations differ only in the rate at which they produce QSMs (with external concentration $a$ ), discussed further below. A QSM can shift a cell from a down-regulated to an up-regulated state with rate constant $\alpha$. Up-regulated cells can down regulate spontaneously (at a rate $\beta$ ). At cell birth, down regulated cells form two down regulated cells and, in view of the complication of whether lux-boxes are occupied in replicated chromosomes, up-regulated cells are taken on average to produce $\gamma$ up-regulated and $2-\gamma$ down-regulated cells for some $\gamma \in[0,2]$. QSMs are produced by both sub-populations (albeit at very different rates), are consumed in the up regulation process and may decay at a rate $\lambda$. For the repression mechanisms we assume the following.

BI mechanism. In Appendix A a simple model of the QSM production process depicted in Figure 2 is derived and studied, from which it is deduced that QSM production occurs at constant rates, namely $\kappa_{d}$ for down-regulated and $\kappa_{u}$ for up-regulated cells (such that $\kappa_{d} \ll \kappa_{u}$ ). Hence, the resulting QSM production terms are the same as for the earlier model. It is also demonstrated in Appendix A, that QSM production is approximately inversely proportional to the concentration of the QSE inhibitor RsmA (or, if QscR is also involved, inversely proportional to a weighted sum of all the QSE inhibitor concentrations); it is assumed, here, that the non-catalytic reaction is very much slower than the catalytic one.

NF mechanism. The negative feedback mechanism will be described by a function $g(a)$, 
such that the resultant QSM production by up-regulated cells is given by $\kappa_{u}(1-g(a))$. The form of function $g(a)$ is discussed below. QSM production by down-regulated cells is assumed not to be affected by negative feedback.

SU mechanism. QSMs are assumed to be soaked up by all cells at a rate $\eta a$.

Finally, we note that depending on the QSM, the QSM is either freely diffuse or actively transported across the cell membrane of a bacterium. In the latter case it has been shown in P. aeruginosa that the internal and external QSM concentrations equilibrate within a few minutes [20], i.e. over a timescale small compared to that of interest; hence only the external concentration needs including. The model studied in this section is therefore

$$
\begin{aligned}
\frac{\mathrm{d} n_{d}}{\mathrm{~d} t} & =r\left(n_{d}+(2-\gamma) n_{u}\right) F\left(n_{d}+n_{u}\right)-\alpha a n_{d}+\beta n_{u}, \\
\frac{\mathrm{d} n_{u}}{\mathrm{~d} t} & =r(\gamma-1) n_{u} F\left(n_{d}+n_{u}\right)+\alpha a n_{d}-\beta n_{u}, \\
\frac{\mathrm{d} a}{\mathrm{~d} t} & =\kappa_{u}(1-g(a)) n_{u}+\kappa_{d} n_{d}-\alpha a n_{d}-\eta a\left(n_{d}+n_{u}\right)-\lambda a,
\end{aligned}
$$

where $F($.$) is a suitable dimensionless bacterial growth function (with F(0)=0$ ) and $r$ is a growth rate constant.

The system (1)-(3) is the same as the model of Ward et al. [26], except for the $g(a)$ function in the QSM source term (NF mechanism) and the QSM soak-up term $\eta n_{T}$ (SU mechanism); we note the BI mechanism is contained in the constants $\kappa_{u}$ and $\kappa_{d}$. The function $g(a)$ describes the NF process, reflecting the promotion of the repressor production by QSMs and its subsequent inhibition of QSM production in up-regulated cells. We assume that $g(a)$ is a smooth, monotonic increasing function of $a$ and we further require that $g(0)=0$ and $g(a) \rightarrow g_{\infty}^{-}$as $a \rightarrow \infty$, where $0<g_{\infty} \leq 1$. We note these constraints on $g(a)$ imply that the maximum and minimum QSM production rates by up-regulated cells are $\kappa_{u}$ and $\kappa_{u}\left(1-g_{\infty}\right)$, respectively; the latter indicates the maximum extent to which QSM production is repressed by negative feedback. The analysis in the later sections will consider general $g(a)$, but for the curve fitting of experimental data described in the next section we chose a simple "S"-shaped function of the simple form

$$
g(a)=g_{\infty} \frac{a^{2}}{a^{2}+a_{c}^{2}}
$$

with constant $a_{c}$, which is expected to have the correct qualitative behaviour. We note that by setting $g_{\infty}=0$ and $\eta=0$ the model of [26] is then recovered.

By adding equations (1) and (2) we have

$$
\frac{\mathrm{d} n_{T}}{\mathrm{~d} t}=r n_{T} F\left(n_{T}\right)
$$

In the analysis which follows we will only be studying the QS process on populations considerably smaller than the carrying capacity $K$, so that growth can be treated as exponential (i.e. $\left.F\left(n_{T}\right) \approx 1\right)$.

\subsection{Experimental work and parameter estimates}

The results of our experimental work and the curve fitting procedure described in Ward et al. [26] are again used as a systematic means of obtaining approximate values for the parameters. We stress that the values presented in the Table 1 serve only as a guide as to the order 
of magnitudes and relative sizes of the dimensionless parameters discussed below, rather than being fundamental rate constants for the phenomena involved. This is due in part to the curve fitting routine converging to a number of local minima, with a wide range of parameter value combinations, but with their sum of squares residues lying within $1 \%$ of each other (the results presented in the table being the apparent global minimum for each of the cases). In view of the analysis described in Section 2.4 and Appendix B, this is not surprising as the combinations of parameters that drives the evolution (and hence the curve fitting) are such that the QSM concentration satisfies

$$
a \approx \frac{k_{u}\left(1-g_{\infty}\right) n_{u}}{(\alpha+\eta) n_{T}-\alpha n_{u}}
$$

during the rapid build up of QSM levels (from (44)) and

$$
a \approx\left\{\begin{array}{cc}
a_{c}\left(\frac{\left(\alpha k_{u}-\beta(\alpha+\eta)\right.}{\left.\beta(\alpha+\eta)-\alpha k_{u}\left(1-g_{\infty}\right)\right)}\right)^{\frac{1}{2}} & g_{\infty}>0, \\
\frac{1}{\alpha \eta}\left(\alpha k_{u}-\beta(\alpha+\eta)\right) & g_{\infty}=0,
\end{array}\right.
$$

during QSM saturation (from (4) and (48)); these combinations of parameters were almost identical for each of the local minima resolved. In any case, the high sensitivity of bacterial behaviour to relatively small variations in environmental conditions, as well as experimental errors, makes "accurate" determination of rate constants impracticable and not particularly instructive; for the purpose of this study order of magnitude approximations suffice. However, while individual parameters are not totally reliable, the expected values of the above relationships are accurate in the appropriate timescales.

The quantities presented in Table 1 are best-fit solutions of experimental data from $P$. aeruginosa strain PAB1 (a clinical burn wound isolate) grown in liquid culture (namely LuriaBertani (LB) broth). The experimental data used here is from a single experiment for which reliable data was obtained during the latter part of the exponential growth phase for both bacterial density and QSM concentration. Consequently, the values shown in Table 1 (particularly for $\alpha$ and $\beta$ ) differ somewhat from those in [26], in which an average of two different experimental runs was taken (one of which was up to 7 hours only). The major discrepancy seems to be that $\beta$ was predicted to be small $\left(<100 \mathrm{hr}^{-1}\right.$ in LB) in the earlier paper, whereby fixing $\beta$ anywhere in the range $0 \leq \beta \leq 100$ yields very poor "best-fit" solutions, failing to capture the saturation of $a$ in the timescale of the experiment. This suggests that the process of "spontaneous" down regulation of up-regulated cells is probably significant, an aspect of quorum sensing our earlier model was unable to confirm. We note, however, generating a best-fit curve of the current data up to $t=4$ hours, fixing $\beta=0$, yielded much more consistent results to those of the earlier paper (cf. the third column with the last column of Table 1 of [26]). In this experiment, exponential growth occurred from zero to about 14 hours and the bacterial population and QSM concentration were taken at roughly hourly intervals. We fitted an exponential growth curve (assuming $F\left(n_{T}\right)=1$ ) to determine $r$ and determined the remainder of the parameters (apart from $\lambda$ ) from the QSM concentration data. The value for $\lambda$ was determined via a separate experiment, whereby the decay rate in LB media was found to be negligibly small; it was, however, found to be non-negligible in other media. Since there is no biological evidence to suggest that the $\mathrm{SU}$ mechanism is relevant for $P$. aeruginosa, we focussed on fitting the data with the NF model, "switching off" $\eta$ by keeping $\eta=0$. However, 


\begin{tabular}{|c|c|c|c|c|c|}
\hline parameter & units & $\begin{array}{l}\text { NF-BI } \\
\text { only case }\end{array}$ & $\begin{array}{c}\text { BI } \\
\text { only case }\end{array}$ & $\begin{array}{c}\text { BI only } \\
\text { with " } \beta=0 "\end{array}$ & $\begin{array}{c}\text { SU-BI } \\
\text { only case }\end{array}$ \\
\hline$K$ & cells $\mathrm{ml}^{-1}$ & $3 \times 10^{10}$ & $3 \times 10^{10}$ & $3 \times 10^{10}$ & $3 \times 10^{10}$ \\
\hline$N_{0}$ & cells $\mathrm{ml}^{-1}$ & $8.2 \times 10^{7}$ & $8.2 \times 10^{7}$ & $8.2 \times 10^{7}$ & $8.2 \times 10^{7}$ \\
\hline$r$ & $\mathrm{hr}^{-1}$ & 0.50 & 0.50 & 0.50 & 0.50 \\
\hline$\kappa_{u}$ & $\mathrm{hr}^{-1}$ & $1.6 \times 10^{7}$ & $1.4 \times 10^{7}$ & $1.1 \times 10^{5}$ & $1.6 \times 10^{7}$ \\
\hline$\kappa_{d}$ & $\mathrm{hr}^{-1}$ & $<1000^{\ddagger}$ & $<1000^{\ddagger}$ & $<1000^{\ddagger}$ & $<1000^{\ddagger}$ \\
\hline$\beta$ & $\mathrm{hr}^{-1}$ & $1.1 \times 10^{7}$ & $1.3 \times 10^{7}$ & 0 & $1.2 \times 10^{7}$ \\
\hline$\lambda$ & $\mathrm{hr}^{-1}$ & 0 & 0 & 0 & 0 \\
\hline$\alpha$ & $\mathrm{ml} \mathrm{hr}^{-1}$ molecule $^{-1}$ & $1.7 \times 10^{-8}$ & $1.3 \times 10^{-7}$ & $9.0 \times 10^{-13}$ & $5.1 \times 10^{-8}$ \\
\hline$\eta$ & $\mathrm{ml} \mathrm{hr}^{-1}$ molecule $^{-1}$ & 0 & 0 & 0 & $2.1 \times 10^{-8}$ \\
\hline$g_{\infty}$ & dimensionless & 0.89 & 0 & 0 & 0 \\
\hline$a_{c}$ & molecule $\mathrm{ml}^{-1}$ & $9.1 \times 10^{13}$ & $\mathrm{n} / \mathrm{a}$ & $\mathrm{n} / \mathrm{a}$ & $\mathrm{n} / \mathrm{a}$ \\
\hline$\gamma$ & dimensionless & $0-2^{\ddagger}$ & 1 & $0.5-2^{\ddagger}$ & $0-2^{\ddagger}$ \\
\hline
\end{tabular}

Table 1: Parameter values determined by curve fitting on experimental data from batch cultures of Pseudomonas aeruginosa grown in liquid LB media. The parameter $N_{0}$ is the initial cell density, i.e. $N_{0}=n_{T}(0)=n_{u}(0)+n_{d}(0)$. The values marked with a $\ddagger$ indicates the range in which the sum of squares lies within approximately $1 \%$ of the minimum value. We note that $\lambda$ was determined to be negligibly small by a separate experiment.

it is unfortunate that the data is not sufficiently refined to determine, through curve fitting means, whether or not the SU mechanism is employed by P. aeruginosa, since consistent curve fits (by which we mean roughly constant sum of squares of the residues) can be obtained for a range of values of $g_{\infty}$ and $\eta$; the final column of Table 1 shows the results for the case in which the NF mechanism is switched off, i.e. $g_{\infty}=0$. Although the curve-fitting results do not preclude the possibility of the SU mechanism being employed, they show that the NF mechanism is sufficient on its own.

For comparison between the current and former models, we determined best-fit parameters for each of the individual QSM repression mechanisms (fitting with data between 0 and 13 hours) and without the NF and SU QSM repressions mechanisms (i.e. $g_{\infty}=0=\eta$, fitting data from 0 to 4 hours). Figure 3 shows the curves generated using these best-fit values (using $\kappa_{d}=1000$ and $\gamma=1$ ) and the experimental data, indicated by the "•"s; the parameters $\kappa_{d}$ and $\gamma$ shown as a range in Table 1 . The Figure shows that, in their respective ranges, all three models agree reasonably with the data. Examination of the figure in more detail suggests that the best fit curve prediction is perhaps weak up to about $t=2$. Here it can be shown that the model predicts an initial growth of QSM concentration proportional to $\exp (r t)$, which generates a slope steeper than the first three data points. There could be a number of reasons for this, experimental error aside and we note that obtaining a best fit curve allowing non-zero $\lambda$ produces a significantly better fit to the data in this range. This is interesting and perhaps suggest that there is a lag time for QSM production by bacteria introduced from an established culture into fresh media. The mechanism for this is not known and further experiments are needed in order to establish the cause of this apparent lag in production; however, the inclusion of non-zero $\lambda$ did not shift significantly the relatively sizes of the other parameters. We note that the parameters common to both the QSM repression and non-QSM repression cases are similar and, as can be seen in Figure 3, the QSM repression processes only become noticeable when the QSM levels saturate. This suggests that, at least in the early stages of colony development, our earlier model provides an adequate description of the QS process. Figure 4 shows the fraction of up-regulated cells against time as predicted 
by the models using the best-fit parameters. The contrast in proportions between the QSM repression and non-repression cases is explained by the analysis of the Section 2.4.

\section{$2.3 \quad$ Non-dimensionalisation}

Before proceeding with the analysis, we non-dimensionalise the system of equations, using the rescalings of Ward et al. [26], namely

$$
t=\hat{t} / r, n_{u}=K \hat{n}_{u}, n_{T}=K \hat{n}_{T}, a=\frac{\kappa_{u} K}{r} \hat{a}, F\left(n_{T}\right)=\hat{F}\left(\hat{n}_{T}\right) ;
$$

where the quantities with hats denotes dimensionless variables. Using (5) we eliminate $n_{d}=$ $n_{T}-n_{u}$ and focus on the following dimensionless system

$$
\begin{aligned}
\frac{\mathrm{d} \hat{n}_{T}}{\mathrm{~d} \hat{t}} & =\hat{n}_{T} \hat{F}\left(\hat{n}_{T}\right), \\
\frac{\mathrm{d} \hat{n}_{u}}{\mathrm{~d} \hat{t}} & =(\gamma-1) \hat{n}_{u} \hat{F}\left(\hat{n}_{T}\right)+\hat{\alpha} \hat{a}\left(\hat{n}_{T}-\hat{n}_{u}\right)-\hat{\beta} \hat{n}_{u}, \\
\frac{\mathrm{d} \hat{a}}{\mathrm{~d} \hat{t}} & =\hat{n}_{u}(1-g(\hat{a}))+\varepsilon\left(\hat{n}_{T}-\hat{n}_{u}\right)-\hat{\mu} \hat{a}\left(\hat{n}_{T}-\hat{n}_{u}\right)-\hat{\eta} \hat{a} \hat{n}_{T}-\hat{\lambda} \hat{a},
\end{aligned}
$$

where $\varepsilon=\kappa_{d} / \kappa_{u}, \hat{\alpha}=\alpha \kappa_{u} K / r^{2}, \hat{\beta}=\beta / r, \hat{\lambda}=\lambda / r, \hat{\eta}=\eta K / r$ and $\hat{\mu}=\alpha K / r$; we assume all parameters in $g(\hat{a})$ are also appropriately non-dimensionalised; for example, in (4) we choose $\hat{a}_{c}=a_{c} r / \kappa_{u} K$, where $\hat{a}_{c}=O\left(10^{-4}\right)$. Using the data in Table 1 the approximate order of magnitudes of the dimensionless parameters are $\hat{\alpha}=O\left(10^{10}\right), \hat{\beta}=O\left(10^{7}\right), \hat{\mu}=O\left(10^{3}\right), \hat{\lambda}$ negligible and $\varepsilon \ll 1$ (seemingly $\varepsilon \lesssim 10^{-4}$ ). The data in the last column of Table 1 suggests that $\eta=O\left(10^{10}\right)$; however, whether or not the $\mathrm{SU}$ is mechanism relevant for this bacterium is uncertain. These dimensionless values contrast somewhat with those given in [26]; however, the relationships of $\varepsilon \ll \hat{\mu} \ll \hat{\beta} \ll \hat{\alpha}$ are maintained. For the remainder of this section the hats on the dimensionless quantities will be dropped for brevity.

\subsection{Asymptotic analysis for exponential growth phase}

\subsubsection{Summary of results}

To gain insights into the governing factors and the role of QSM repression processes in QS, we seek solutions of the model in the biologically motivated limit $\varepsilon \rightarrow 0$. We assume a small initial population of bacteria and investigate the sequence of important timescales involved in the evolution of $n_{u}$ and $a$ during the exponential growth phase of the population. The system studied is

$$
\begin{aligned}
n_{T} & =N_{0} \mathrm{e}^{t}, \\
\frac{\mathrm{d} n_{u}}{\mathrm{~d} t} & =(\gamma-1-\beta) n_{u}+\alpha a\left(N_{0} \mathrm{e}^{t}-n_{u}\right), \\
\frac{\mathrm{d} a}{\mathrm{~d} t} & =n_{u}(1-g(a))+(\varepsilon-\mu a)\left(N_{0} \mathrm{e}^{t}-n_{u}\right)-\eta a N_{0} \mathrm{e}^{t}-\lambda a,
\end{aligned}
$$

subject to $n_{u}(0)=0$ and $a(0)=0$, in the limits of $\varepsilon \rightarrow 0$ and $N_{0} \rightarrow 0$. The details of the asymptotic analysis is given are Appendix B and the main results demonstrating the effects of QSM repression are discussed below; the large-time behaviour is summarised in Table 2 . The analysis draws out a crucial parameter, namely

$$
\Theta=\frac{\alpha}{\mu+\eta}-(\beta+2-\gamma)
$$




\begin{tabular}{|c|c|c|c|c|}
\hline \multirow[b]{2}{*}{$\Theta$} & \multirow[b]{2}{*}{ notes } & \multicolumn{2}{|r|}{$t \rightarrow \infty$ behaviour } & \multirow[b]{2}{*}{ SS } \\
\hline & & $n_{u} \sim$ & $a \sim$ & \\
\hline \multirow[t]{2}{*}{$\Theta>0$} & $\eta>0$ & $\frac{\alpha a^{*}}{\alpha a^{*}+\beta+2-\gamma} n_{T}$ & 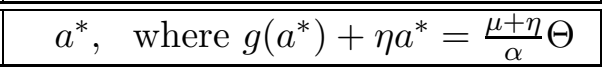 & $* *$ \\
\hline & $\begin{array}{l}g_{\infty}>\frac{\mu \Theta}{\alpha} \\
g_{\infty}=\frac{\mu \Theta}{\alpha} \\
g_{\infty}<\frac{\mu \Theta}{\alpha}\end{array}$ & $\begin{array}{c}\frac{\alpha a^{*}}{\alpha a^{*}+\beta+2-\gamma} n_{T} \\
n_{T} \\
n_{T} \\
\end{array}$ & $\begin{array}{c}a^{*}=g^{-1}(\mu \Theta / \alpha) \\
\text { sub-exponential }^{\dagger} \\
\frac{\mu \Theta-\alpha g_{\infty}}{\alpha(1+\lambda)} n_{T} \\
\end{array}$ & \begin{tabular}{l|}
$* *$ \\
- \\
-
\end{tabular} \\
\hline$\Theta=\varepsilon^{\delta} \theta$ & $\begin{aligned} g(a) \sim B a^{m \ddagger} & m>1: \delta=\frac{1}{2} \\
m & =1: \delta=\frac{1}{2} \\
m<1: \delta & =\frac{m}{m+1}\end{aligned}$ & $\begin{array}{l}\varepsilon^{\frac{1}{2}}(\mu+\eta) a n_{T} \\
\varepsilon^{\frac{1}{2}}(\mu+\eta) a n_{T} \\
\varepsilon^{\frac{1}{m+1}}(\mu+\eta) a^{*} n_{T} \\
\end{array}$ & \begin{tabular}{|c|}
$\varepsilon^{\frac{1}{2}} \frac{(\eta+\mu)^{2} \theta+\sqrt{\theta^{2}(\mu+\eta)^{4}+4 \alpha^{2} \eta(\mu+\eta)}}{2 \alpha \eta(\mu+\eta)}$ \\
$\varepsilon^{\frac{1}{2}} \frac{(\eta+\mu)^{2} \theta+\sqrt{\theta^{2}(\mu+\eta)^{4}+4 \alpha^{2} \eta(B+\mu+\eta)}}{2 \alpha \eta(B+\mu+\eta)}$ \\
$\varepsilon^{\frac{1}{m+1}} a^{*}$, where $a^{* m+1}-\frac{\theta(\mu+\eta)}{B \alpha} a^{*}=\frac{1}{B(\mu+\eta)}$
\end{tabular} & $\begin{array}{l}* * \\
* * \\
* * \\
\end{array}$ \\
\hline$\Theta<0$ & & 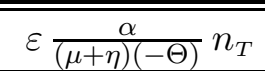 & $\bar{\varepsilon} \frac{\alpha+(\mu+\eta)(-\Theta)}{(\mu+\eta)^{2}(-\Theta)}$ & $*$ \\
\hline
\end{tabular}

Table 2: A summary of large-time solutions of (27) and (28) in the limit of $\varepsilon \rightarrow 0$ derived from the asymptotic analysis described in Appendix B. The case in which saturation of QSM levels occur has been labelled with a "*" in the SS column, while those labelled with "**"s have saturated due to a QSM repression mechanism. 'Growth is dependent on the behaviour of $g(a)$ as $a \rightarrow \infty$; ${ }^{\ddagger}$ is the assumed behaviour of $g(a)$ as $a \rightarrow 0^{+}$, where $B$ and $m$ are positive constants.

which, in common with the constant $\Theta$ of Ward et al. [26] (i.e. the same quantity with $\eta=0$ ), governs many aspects of the QS process during exponential population growth.

The analysis of Appendix B shows that substantial up regulation can be achieved only when $\Theta>0$, in agreement with our earlier model. The consideration of general $N_{0}$ changes only the timescale of the initial, slow up regulation, phase from $t=\ln (1 / \varepsilon)+O(1)$ in $[26]$ to $t=$ $\ln \left(1 / N_{0}\right)+O(1)$; the period for rapid up regulation, $t=\ln (1 / \varepsilon) / \Theta+O(1)$, remains unchanged. This suggests that when a rapid jump in QS behaviour occurs then $\ln (1 / \varepsilon) / \Theta \ll \ln \left(1 / N_{0}\right)$. These important timescales, leading to the large-time solutions in Table 2, are principally affected by the BI and SU processes $(\eta>0)$ and not by NF; this is discussed in more detail in Section 2.4.2. Only in the later timescales, if the QSMs have reached a sufficient concentration, does the NF mechanism significantly influence the evolution of $a$ and $n_{u}$. In [26] it was shown for $\Theta \geq 0$ that as $t \rightarrow \infty, n_{u} \sim n_{T}$ and QSM levels grow exponentially. The table shows that $\eta>0$ is sufficient for the experimentally observed saturation of QSM concentration to occur. In the case of no direct repression, $\eta=0$, the situation is more complicated as the large time behaviour is governed by the strength of the NF process, i.e. by the size of $g_{\infty}$. When feedback is weak $\left(g_{\infty}<\mu \Theta / \alpha\right)$ all cells (to leading order) will eventually be up-regulated, with QSM levels increasing exponentially (the behaviour being similar to that predicted by our earlier model). For a sufficiently strong negative feedback response (i.e. $g_{\infty}>\mu \Theta / \alpha$ ), QSM saturation results at large time. Moreover, both NF (if $g_{\infty}>\mu \Theta / \alpha$ ) and SU repression mechanisms reduces the large time up-regulated cell fraction, so that $1-n_{u} / n_{T}=O(1)$ rather than $n_{u} / n_{T} \sim 1$. Negligible up-regulation occurs for $\Theta<0$ and for $0<\Theta \ll 1$ and there is no noticeable negative feedback response in the case of $\Theta<0$. In Ward et al. [26] it was shown that when $\Theta=0$ complete up-regulation of the population eventually occurs; however, in the current case either the NF or SU repression mechanisms will inhibit QSM production sufficiently for up regulation to remain negligible in this borderline case. 


\subsubsection{Implications of analysis for quorum sensing repression}

In this section we briefly discuss the effect of the repression mechanisms on QS by investigating the timescale for up regulation and the ultimate up-regulated cell fraction using the dimensional form of the parameters; this facilitates the study of the role of the BI mechanism in particular. It is assumed that the parameters are such that substantial up regulation occurs i.e., from (12),

$$
\Theta\left(R_{0}, \eta\right)=\frac{\alpha \kappa_{u}\left(R_{0}\right)}{r(\alpha+\eta)}-\frac{\beta}{r}-(2-\gamma)>0
$$

where $\kappa_{u}\left(R_{0}\right)$ is given by (39), $R_{0}$ is the concentration of the QSE inhibitor, and all parameters are dimensional; the functional form for $\Theta\left(R_{0}, \eta\right)$ is convenient for the discussion below. We note that since $\mathrm{d} \kappa_{u}\left(R_{0}\right) / \mathrm{d} R_{0}<0$ (from (39)) then $\partial \Theta / \partial R_{0}<0$ and $\partial \Theta / \partial \eta<0$, i.e. over expressing either the $\mathrm{BI}$ or $\mathrm{SU}$ mechanisms may result in near complete inhibition of up regulation. From the analysis in Appendix B, the timescale $T_{E}$ on which substantial up regulation occurs is given by

$$
T_{E}\left(R_{0}, \eta\right) \sim \frac{1}{r} \ln \left(\frac{K}{n_{T}(0)}\right)+\frac{1}{r \Theta\left(R_{0}, \eta\right)} \ln \left(\frac{\kappa_{u}\left(R_{0}\right)}{\kappa_{d}\left(R_{0}\right)}\right),
$$

and the up-regulated cell fraction $N_{E}^{*}$ is

$$
N_{E}^{*}\left(R_{0}, \eta, g_{\infty}\right)=\frac{n_{u}}{n_{T}} \sim \frac{\alpha a_{E}^{*}\left(R_{0}, \eta, g_{\infty}\right)}{\alpha a_{E}^{*}\left(R_{0}, \eta, g_{\infty}\right)+\beta+r(2-\gamma)},
$$

where $a_{E}^{*}\left(R_{0}, \eta, g_{\infty}\right)$ satisfies

$$
g\left(a_{E}^{*}\right)+\frac{\eta}{\kappa_{u}\left(R_{0}\right)} a_{E}^{*}-\frac{r(\alpha+\eta)}{\alpha \kappa_{u}\left(R_{0}\right)} \Theta\left(R_{0}, \eta\right)=0 .
$$

We recall that the parameters $R_{0}, g_{\infty}$ and $\eta$ control the strength of the BI, NF and SU mechanisms, respectively, and their effects are as follows.

BI mechanism. For general parameter values it is not possible to determine the sign of $\partial T_{E} / \partial R_{0}$. However, in the biologically appropriate regime $k_{d} \ll k_{u}$, we have $\partial T_{E} / \partial R_{0}>$ 0 ; hence increasing the QSE inhibitor concentration will, as is to be expected, prolong the timescale for up regulation (this is in agreement with the experimental results of Chugani et al. [6]). Furthermore, we have $\partial a_{E}^{*} / \partial R_{0}<0$ and $\partial N_{E}^{*} / \partial R_{0}<0$, so increasing $R_{0}$ reduces QSM build up and the up-regulated cell fraction. Moreover, in the absence of QSE inhibitor (i.e. for $R_{0}=0$ ) the modelling of Appendix A predicts there will be no saturation of QSE levels, resulting with the $\kappa_{d}$ and $\kappa_{u}$ being linear functions of time rather than constant; this complicates the modelling, substantially, since the time dependence of the mRNA-lasI and QSE concentrations would also have to be explicitly considered. In the limit $R_{0} \rightarrow 0$, however, $T_{E} \sim \ln \left(K / n_{T}(0)\right) / r$ and $N_{E}^{*} \sim 1$ (since $\left.a_{E}^{*} \rightarrow \infty\right)$, implying a QSM production run away even if the NF or SU mechanisms are in operation. Although QSE inhibition is necessary for the experimentally observed saturation of QSM concentration, it is not sufficient (see [26]), and we need either the $\mathrm{NF}$ or the SU mechanism to be operating also in order to predict it.

NF mechanism. This mechanism does not affect the timescale for up regulation; however, $\partial a_{E}^{*} / \partial g_{\infty}<0$ and $\partial N_{E}^{*} / \partial g_{\infty}<0$, and hence the up-regulated cell fraction decreases with increased intensity of negative feedback. 
SU mechanism. Since $\partial \Theta / \partial \eta<0$ and $\partial a_{E}^{*} / \partial \eta<0$ then $\partial T_{E} / \partial \eta>0$ and $\partial N_{E}^{*} / \partial \eta<0$, i.e. increasing the rate of soaking up of QSMs increases the timescale for up regulation and reduces the up-regulated cell fraction. We note, however, that the effects of the SU mechanism will only be significant if $\eta \geq O(\alpha)$.

\subsection{Implications to therapy}

It is widely thought that attacking the quorum sensing process is a viable approach in the development of new therapies. Such strategies under current investigation include the development of agents that soak up freely diffusing QSMs or interfere with the QSP process in some way, thus preventing up regulation and virulence; the mathematical modelling of such therapies is currently being undertaken [1]. Motivated by the prediction that only a fraction of a population will become up-regulated when $\Theta>0$ and either the NF or SU mechanism is operating, we investigate the effectiveness of a (putative) drug that targets bacteria expressing a particular gene during virulence. For example, it is well known that sessile bacteria in biofilms express genes that enable greater drug resistance than planktonic ones, even though quorum sensing is not involved here, the hypothesis seems feasible. The fact that only a fraction of a population is up-regulated will therefore have significant consequences on the effectiveness of the drug. In this section we briefly discuss a simple extension to the model to describe the action of such as drug in an experimental system such as that described in Section 2.2.

We assume that the up-regulated cell targetting drug (concentration $w$ ) is contained in the culture medium at the start of population growth and it kills only those cells that are up regulated. In dimensionless form the model studied is

$$
\begin{aligned}
\frac{\mathrm{d} n_{T}}{\mathrm{~d} t} & =n_{T}-w n_{u}, \\
\frac{\mathrm{d} n_{u}}{\mathrm{~d} t} & =(\gamma-1-\beta-w) n_{u}+\alpha a\left(n_{T}-n_{u}\right), \\
\frac{\mathrm{d} a}{\mathrm{~d} t} & =n_{u}(1-g(a))+(\varepsilon-\mu a)\left(n_{T}-n_{u}\right)-\eta a n_{T}-\lambda a, \\
\frac{\mathrm{d} w}{\mathrm{~d} t} & =-\delta w n_{u},
\end{aligned}
$$

subject to the initial conditions $n_{T}(0)=N_{0}, n_{u}(0)=0, a(0)=0$ and $w(0)=w_{0}$. We note that $w$ has been scaled with the cell kill rate, rather than the initial drug concentration, in order to make explicit the effects of the drug concentration discussed below. The dimensionless parameter $\delta$ governs the amount of drug consumed during the cell killing process. It has also been assumed that over the timescale of interest there is negligible degradation or loss of the drug in the medium. The issues, therefore, concern how the strength of the repression mechanism governs the effectiveness of a drug given an initial dose.

The purely hypothetical nature of the drug does not warrant detailed description of the mathematical analysis that can be undertaken on the model. It is worth noting, however, that substantial up-regulation and consequently significant drug induced killing only occur when $\Theta_{w}>0$, where

$$
\Theta_{w}=\frac{\alpha}{\mu+\eta}-\left(\beta+2-\gamma+w_{0}\right)
$$

For $\Theta_{w}>0$ there are two contrasting large-time outcomes. 
1. Eventual consumption of the drug, leading ultimately to a resumption of exponential population growth (and exponential decay of the drug). This outcome requires $\delta>0$.

2. Sufficient killing of the population that the population ultimately declines exponentially, with the drug tending to a steady-state, $w \rightarrow w^{*}$ say (note if $\delta=0$ then $w^{*}=w_{0}$ ).

It can be shown that a necessary condition for scenario 2 . is that $w_{0} \geq 1$.

Figure 5 demonstrates the two large-time outcomes listed above. Here $\delta=10$, with the other parameters determined from Table 1 in dimensionless form. The bifurcation between the two outcomes occurs at $w_{0} \approx 16.74$. We observe that for $w_{0}=16$ there is insufficient drug to kill off the population, although the dose is sufficiently strong to induce a decline between $t=5$ and $t=20$. The drug concentration seems to decay slowly until about $t=40$ when there is a very rapid drop; this corresponds to recovery of growth of the form described in Appendix B.2.3 leading to $w \sim W_{c} \exp \left(-\mathrm{e}^{(\Theta+1) t}\right.$ ) (for some constant $W_{c}^{*}$ ) during this phase. For $w_{0}=17$ there is sufficient drug that, following an initial non-negligible level of consumption whilst the population density is of $O(0.1)$, enough remains to prevent population recovery and outcome 2. above ensues. The effects of the NF repression mechanism on the effectiveness of a drug dose is demonstrated in Figure 6 for various $g_{\infty}$ by plotting the bifurcation curves between the two large-time outcomes in $\delta-w_{0}$ space. The curves are computed by a systematic process of trial and error, employing a bisection technique. In all cases, as expected, increasing the drug consumption rate $\delta$ requires more drug initially if the the population is to be eliminated. Moreover, increasing the intensity of the NF mechanism leads to significantly more drug being required in order for the therapy to be effective.

\section{Role of QSM repression in developing biofilms}

\subsection{Mathematical model}

In this section we extend the simple model for QS in early biofilms described in Ward et al. [25], the conclusions drawn also being relevant to the saturated batch culture case described in Section 4 of [26]. The detailed derivation of the model is given in [25] and here we give only an outline. We assume that the biofilm is homogenous (i.e with uniform bacterial density), densely populated and with a very small depth to width ratio (i.e. $H / L \ll 1$ ). We exploit $H / L \ll 1$ and apply thin-film theory so that explicit consideration of depth variations can be avoided and, through integrating over depth, we instead consider cross-sectional cell densities and QSM concentration. The colony consists of down-regulated (cross-sectional density $N_{d}$ ) and up-regulated (cross-sectional density $N_{u}$ ) cells, such that the total local cross-sectional density is given by $N_{T}=N_{d}+N_{u}$; we note that since the colony is homogenous we have $H=\omega N_{T}$, where constant $\omega$ is the mean volume that a cell occupies. The process of cell growth creates volume within the biofilm, which generates movement described by a velocity field, which in two dimensions is given by $\boldsymbol{v}=(v, w)$, where $v$ and $w$ are the tangential and normal velocities with respect to a (flat) substrate. The application of thin-field theory reduces the problem to one in which only the tangential velocity $v$ is present. Defining $a$ to be the local QSM concentration we find by applying thin-film assumptions that the cross-sectional concentration of QSM $(A)$ is given by $A=a H=a \omega N_{T}$. For simplicity, the effective direction of biofilm growth is governed by a prescribed function $\phi\left(N_{T}\right)$; however, in the analysis to follow we will not be considering growth in any detail. The assumptions described in Section 
2.1 on the various aspects of QS again apply, but we assume that QSMs can also escape from through the surface of the biofilm, hence being permanently lost from the system, at a rate proportional to $Q / N_{T}$. The QSM repression processes will be modelled as before, again using the negative feedback function $g(a)=G\left(A / \omega N_{T}\right)$ introduced in Section 2.1. Although the choice is not significant in the analysis to follow, we will adopt either Cartesian or cylindrical geometry, whereby the biofilm is constrained within a moving boundary, at location $x=S(t)$ in Cartesian or $\rho=S(t)$ in cylindrical geometry, the velocity $v$ being, respectively, in the $x$ and $\rho$ directions. For brevity we present the model in dimensionless form, the scalings being analogous to those of Section 2.3 and being detailed in Ward et al. [25]; in summary, $x$ or $\rho$ is scaled with the initial biofilm radius $S(0)=S_{0}$, time with the reciprocal of the growth rate $r$ and $N_{i}$ with the cross-sectional carrying capacity $K_{s}$ (i.e. the maximum number of cells per unit area), giving for the Cartesian case

$$
\begin{aligned}
\frac{\partial N_{T}}{\partial t}+\frac{\partial}{\partial x}\left(v N_{T}\right) & =N_{T} F\left(N_{T}\right) \\
\frac{\partial N_{u}}{\partial t}+\frac{\partial}{\partial x}\left(v N_{u}\right) & =(\gamma-1) N_{u} F\left(N_{T}\right)+\alpha A \frac{\left(N_{T}-N_{u}\right)}{N_{T}}-\beta N_{u}, \\
\frac{\partial A}{\partial t}+\frac{\partial}{\partial x}(v A)= & D \frac{\partial^{2} A}{\partial x^{2}}+\varepsilon\left(N_{T}-N_{u}\right)+N_{u}\left(1-G\left(A / N_{T}\right)\right) \\
-A\left(\mu \frac{\left(N_{T}-N_{u}\right)}{N_{T}}+\hat{\eta}+\lambda+\frac{Q}{N_{T}}\right), & \\
\frac{\partial v}{\partial x} & =N_{T} \phi\left(N_{T}\right) F\left(N_{T}\right),
\end{aligned}
$$

where $N_{T}=N_{d}+N_{u}$. For the cylindrical geometry case we replace the $\partial(\cdot) / \partial x$ terms with $\left(1 / \rho^{2}\right) \partial\left(\rho^{2} \cdot\right) / \partial \rho$ and $\partial^{2}(\cdot) / \partial x^{2}$ with $\left(1 / \rho^{2}\right) \partial\left(\rho^{2} \partial(\cdot) / \partial \rho\right) / \partial \rho$. We note that the dimensionless quantity $\hat{\eta}=\eta / \omega r$, where $\eta$ is the same quantity as in Section 2; we will drop the hat in the remainder of this section.

The dimensionless functions $F\left(N_{T}\right)$ and $\phi\left(N_{T}\right)$ govern the growth rate and direction, respectively, and are chosen so that $F(1)=0$ and $\phi(1)=1$. These reflect limitations of nutrient diffusion in more developed biofilms, restricting the net direction of growth to be tangential to the surface as $N_{T} \rightarrow 1$. However, in the analysis that follows we will assume, for simplicity, that $N_{T}=1$, i.e. we will be investigating the QS process on a local region, away from the biofilm edge, at which the biofilm has grown to its full capacity and $A$ is independent of $x$ in the relevant regime.

The behaviour of sessile bacteria in biofilms can be very different to that of their planktonic counterparts in liquid media. Thus, the kinetics predicted from results using batch cultures, where the bacteria are planktonic, may not be applicable in biofilm situations. Obtaining parameter values from experiments using biofilms is difficult and has not as yet been accomplished.

\subsection{Asymptotic analysis for fixed depth biofilm}

In this section we investigate QS in a fixed depth biofilm in which there is no net growth, starting with no up-regulated cells and zero QSM concentration. We assume that $N_{T}=1$ and that (23)-(25) is subject to

$$
\begin{array}{ll}
t=0, & N_{u}=0, A=0, \\
x=0, & v=0, \frac{\partial A}{\partial x}=0,
\end{array}
$$




\begin{tabular}{|c|c||c|c|}
\hline \multirow{2}{*}{$\Psi_{b}$} & \multicolumn{2}{|c||}{ notes } & \multicolumn{2}{c|}{$t \rightarrow \infty$ behaviour } \\
\cline { 3 - 4 } & & $N_{u} \sim$ & $A \sim$ \\
\hline \hline$\Psi_{b}>0$ & & $\frac{\alpha A^{*}}{\alpha A^{*}+\beta}$ & $A^{*}$, where $G\left(A^{*}\right)=\frac{\Psi_{b}}{\alpha}-(\eta+\lambda+Q) A^{*}$ \\
\hline \hline$\Psi_{b}=\varepsilon^{\sigma} \psi$ & ${ }^{\dagger} G(A) \sim B A^{m}: m>1 ; \sigma=\frac{1}{2}$ & $\frac{\alpha}{\beta} A$ & $\varepsilon^{\frac{1}{2}} \frac{1}{2} \frac{\psi+\sqrt{\psi^{2}+4 \alpha \beta(\eta+\lambda+Q)}}{\alpha(\eta+\lambda+Q)}$ \\
\hline & $m=1 ; \sigma=\frac{1}{2}$ & $\frac{\alpha}{\beta} A$ & $\varepsilon^{\frac{1}{2}} \frac{1}{2} \frac{\psi+\sqrt{\psi^{2}+4 \alpha \beta(B+\eta+\lambda+Q)}}{\alpha(B+\eta+\lambda+Q)}$ \\
\hline & $m<1 ; \sigma=\frac{m}{m+1}$ & $\frac{\alpha}{\beta} A^{*}$ & $\varepsilon^{\frac{1}{m+1}} A^{*}$, where $B A^{* m+1}=\frac{\psi}{\alpha} A^{*}+\frac{\beta}{\alpha}$ \\
\hline \hline$\Psi_{b}<0$ & & $\varepsilon \frac{\alpha}{\left(-\Psi_{b}\right)}$ & $\varepsilon \frac{\beta}{\left(-\Psi_{b}\right)}$ \\
\hline
\end{tabular}

Table 3: Summary of large time solutions of (27) and (28) in the limit of $\varepsilon \rightarrow 0$ derived from the asymptotic analysis described in Appendix C. ${ }^{\dagger}$ denotes the behaviour of $G(A)$ as $A \rightarrow 0$.

(in cylindrical geometry we instead have $\partial A / \partial \rho=0$ at $\rho=0$ ). We note that considering $N_{T}(0)=N_{0}<1$ instead simply delays the timescale for up regulation (if it occurs), for example by $t=O\left(\ln \left(1 / N_{0}\right)\right)$ for the logistic growth case (i.e. $\left.F\left(N_{T}\right)=1-N_{T}\right)$. It is also assumed that the biofilm radius $S(t)$ is sufficiently large (i.e. $D / S^{2} \ll 1$ ) that QSM loss is restricted to a boundary layer, of size $\sqrt{D} / S \ll 1$, at the biofilm rim and has negligible effect on the behaviour over the bulk of the biofilm; this assumption generates spatial uniformity, with $\partial^{2} A / \partial x^{2}$ negligible, away from the boundary layer. We thus simplify the analysis by writing $N_{u}(x, t)=N_{u}(t)$ and $A(x, t)=A(t)$. Applying these assumptions the equations studied are

$$
\begin{aligned}
\frac{\mathrm{d} N_{u}}{\mathrm{~d} t} & =\alpha A\left(1-N_{u}\right)-\beta N_{u}, \\
\frac{\mathrm{d} A}{\mathrm{~d} t} & =N_{u}(1-G(A))+\varepsilon\left(1-N_{u}\right)-\mu A\left(1-N_{u}\right)-(\eta+\lambda+Q) A,
\end{aligned}
$$

subject to $N_{u}(0)=A(0)=0$, as $\varepsilon \rightarrow 0$.

The asymptotic analysis of (26)-(28) is described in Appendix C and, as with Ward et $a l .[25]$, a parameter crucial arises for quorum from the analysis, namely

$$
\Psi_{b}=\alpha-\beta(\eta+\lambda+\mu+Q)
$$

$\Psi_{b}>0$ is required for substantial up regulation to occur. Table 3 show the large time solutions of $N_{u}$ and $A$ for the various regimes of $\Psi_{b}$ and forms of the negative feedback function $G(A)$. In common with the exponentially growing case, if substantial up regulation occurs (i.e. if $\left.\Psi_{b}>0\right)$ then the timescale for this is influenced only by the BI and SU mechanisms and not by the NF mechanism. The effect of the NF mechanism is only non-negligible for large time if $\Psi_{b}>0$ or $\left|\Psi_{b}\right|$ is small (provided that $G(A) \rightarrow 0$ sufficiently slowly as $A \rightarrow 0$, i.e. when $m \leq 1)$. In comparison to the exponentially growing population case, the effects of the QSM repression mechanisms are less pronounced in the biofilm case, having little effect on the qualitative behaviour of the evolution of $N_{u}$ and $A$. (We note this is also true for a saturated population in batch cultures described in Section 4 of [26]). 


\subsubsection{Implications of analysis for quorum sensing repression in biofilms}

As in Section 2.4.2, the effects of the repression mechanisms on the timescale for up regulation and on the up-regulated cell fraction are now studied using the dimensional form of the parameters. Again we assume that substantial up regulation occurs, so that

$$
\Psi_{b}\left(R_{0}, \eta\right)=\frac{\alpha}{\omega r^{2}}\left(\kappa_{u}\left(R_{0}\right)-\beta\right)-\frac{\beta}{\omega r^{2}}\left(\eta+\omega \lambda+\frac{Q}{K_{s}}\right)>0 .
$$

where $\kappa_{u}\left(R_{0}\right)$ is given by (39). Similarly to $\Theta\left(R_{0}, \eta\right)$ in Section 2.4.2, $\Psi_{b}\left(R_{0}, \eta\right)$ is monotonic decreasing in both $R_{0}$ (since $\left.\mathrm{d} \kappa_{u}\left(R_{0}\right) / \mathrm{d} R_{0}<0\right)$ and $\eta$; hence increasing either parameter will reduce the parameter ranges for possible up regulation. Assuming the biofilm depth has reached a steady-state, the timescale $T_{B}$ for up regulation is

$$
T_{B}\left(R_{0}, \eta\right) \sim \frac{1}{\omega_{+}\left(R_{0}, \eta\right)} \ln \left(\frac{\kappa_{u}\left(R_{0}\right)}{\kappa_{d}\left(R_{0}\right)}\right)
$$

where

$$
\begin{aligned}
\omega_{+}\left(R_{0}, \eta\right)=-\frac{1}{2 r \omega}(\alpha & \left.+\eta+\omega(\lambda+\beta)+\frac{Q}{K_{s}}\right) \\
& +\frac{1}{2 r \omega}\left(\left(\alpha+\eta+\omega(\lambda-\beta)+\frac{Q}{K_{s}}\right)^{2}+4 \omega \kappa_{u}\left(R_{0}\right) \alpha\right)^{\frac{1}{2}} .
\end{aligned}
$$

The fraction of $N_{B}^{*}$ of up-regulated cells is given by

$$
N_{B}^{*}\left(R_{0}, \eta, g_{\infty}\right) \sim \frac{\alpha A_{B}^{*}\left(R_{0}, \eta, g_{\infty}\right)}{\alpha A_{B}^{*}\left(R_{0}, \eta, g_{\infty}\right)+\beta \omega K_{s}}
$$

where $A_{B}^{*}\left(R_{0}, \eta, g_{\infty}\right)$ satisfies

$$
G\left(A_{B}^{*}\right)+\frac{\left(K_{s}(\eta+\omega \lambda)+Q\right)}{\kappa_{u}\left(R_{0}\right) K_{s}^{2} \omega} A_{B}^{*}-\frac{\omega r^{2}}{\kappa_{u}\left(R_{0}\right) \alpha} \Psi_{b}\left(R_{0}, \eta\right)=0 .
$$

The effects of each of the repression mechanisms are similar to the exponential case discussed in Section 2.4.1 and can be summarised as follows.

BI mechanism. It can be shown that $\partial N_{B}^{*} / \partial R_{0}<0$ and, by virtue of $\kappa_{d} \ll \kappa_{u}$, that $\partial T_{B} / \partial R_{0}>0$. Hence, increasing the concentration of the QSE inhibitor $R_{0}$, results in the expected response of increasing the timescale for up regulation and decreasing the large time up-regulated cell density. Moreover, the limit $R_{0} \rightarrow 0$ results in $T_{B} \rightarrow 0$ and $N_{B}^{*} \rightarrow 1$ (since $A_{B}^{*} \rightarrow \infty$ ) so, regardless of the other repression mechanisms operating, the absence of QSE inhibition will lead to the run away of QSM production and, ultimately, up regulation of all cells in the population.

NF mechanism. Negative feedback again does not affect the timescales of up regulation, but since $\partial N_{B}^{*} / \partial g_{\infty}<0$ the up-regulated cell fraction decreases with increasing strength of the feedback response.

SU mechanism. It can be shown that $\partial \omega_{+} / \partial \eta<0$ and $\partial A_{B}^{*} / \partial \eta<0$ so that $\partial T_{B} / \partial \eta>0$; hence, as expected, increasing the rate of soaking up of QSMs leads to an increase in the timescale for up regulation and a reduced steady-state up-regulated cell fraction. 


\subsection{A note on the role of QSM repression on travelling wave behaviour}

In Ward et al. [25] it was shown that, in the limit case $\varepsilon=0$, equations (23)-(25) can exhibit travelling wave behaviour for large time, whereby a wave front advances from the steady-state solutions $\left(N_{u}^{*}, A^{*}\right)$, given by $(71)$, towards the trivial solution $\left(N_{u}=A=0\right)$. The analysis involved translating the equations to a travelling wave co-ordinate $z$ using $\sqrt{D} z=x-P(t)$, where $P(t) \sim C t$ as $t \rightarrow \infty$ is the position of the front, such that $N_{u} \rightarrow 0$ and $A \rightarrow 0$ as $z \rightarrow+\infty$. It was shown that travelling wave solutions can only exist if $\Psi_{b}>0, \varepsilon=0$ and $N_{T} \equiv 1$. The wave speed $c=C / \sqrt{D}$ can be determined by applying linearisation of the equations in the limit $z \rightarrow+\infty$, with valid solutions existing for all $c$ lying in the range $0<c_{\min } \leq c<\infty$ (as with Fisher's equation); however, for general sufficiently rapidly decaying initial conditions, $c=c_{\min }$ is as usual the observed wave speed. The equivalent analysis for the current model proceeds in exactly the same way, whereby defining $c_{\min }$ such that

$$
\Omega\left(c_{\text {min }}\right) \equiv 4 \Upsilon\left(c_{\text {min }}\right)-27 \Phi\left(c_{\text {min }}\right)=0,
$$

where

$$
\begin{aligned}
& \Upsilon(c)=\frac{1}{3}\left(c-\frac{\beta}{c}\right)^{2}+\beta+\mu+\eta+\lambda+Q, \\
& \Phi(c)=\frac{\Psi_{b}}{c}-\frac{2}{27}\left(c-\frac{\beta}{c}\right)^{3}-\frac{1}{3}(\beta+\mu+\eta+\lambda+Q)\left(c-\frac{\beta}{c}\right),
\end{aligned}
$$

implies valid (non-negative) solutions have $c \geq c_{\min }$. We note that since the negative feedback term is nonlinear and that $G(0)=0$, this process has no effect on the wave speed so long as linear selection of $c_{\text {min }}$ pertains. However, by virtue of (71), the cell density and QSM concentration behind the wave front are affected. We note also that by differentiating $\Omega\left(c_{\min }(\eta) ; \eta\right)$ with respect to $\eta$ and noting from [25] that $\partial \Omega(c) / \partial c>0$, it can be shown that $\partial c_{\min } / \partial \eta<0$, i.e. the wave speed decreases with increasing strength of inhibition of QSM production. It can also been shown, by reverting to dimensional quantities, that (the dimensional) speed $c_{\text {min }}$ decreases with $R_{0}$, so repression by the BI mechanism reduces the wave speed.

\section{Discussion}

In this paper, two existing models have been extended to investigate in detail the role of three experimentally observed QS repression mechanisms within exponentially growing colonies and developing biofilms. The mechanisms investigated involve QSE inhibitors (e.g. RsmA molecules in $P$. aeruginosa), negative feedback (e.g. RsaL in P. aeruginosa) and constitutively produced QSM soaking up molecules (e.g. TrlR in A. tumefaciens ). Although the main focus has been on the P. aeruginosa and A. tumefaciens QS systems, it is likely that there are equivalent QS repression systems in many species of bacteria both Gram positive and negative, to which the modelling in this paper should also apply. Using curve fitting techniques we determined estimates of the model parameters, which lead to good fits with experimental data. Moreover, the model predicts that either NF or SU is sufficient to obtain the experimentally observed saturation of QSM concentration during the exponential growth phase. We stress that only some of the parameter estimates can be regarded as reliable (namely $K, r$ and $\lambda$, from independent data, and $\kappa_{u}, \alpha$, and $\beta$ from time-course QSM concentration data), as 
these are the dominant parameters governing the evolution of QSM concentration during this growth phase. Interestingly, the need for non-negligible value for $\beta$ predicted by the best-fits with the current model suggests that turnover in the attachment of the QSP-QSM complex to the lux-box is a significant process in the dynamics of quorum sensing.

The modelling predicts that the repression mechanisms have most impact on bacterial populations during the exponential phase of growth, most notably on the eventual up-regulated cell fraction and on the saturation levels of QSM concentration (provided that $g_{\infty}>\mu \Theta / \alpha$ ). It seems that the main advantage to the bacteria of the QS repression processes is the prevention of a superfluous runaway of QSM production. Moreover, it is of no benefit for the entire population of cells to be up-regulated when only a fraction needs to be active. It is interesting that the simulations shown in Figure 4, using the best-fit parameters, predict that only about $10-20 \%$ of the population will be up-regulated; from this it can be supposed that nutrient expenditure by QS is a relatively small proportion of that which would occur in a system without QSM repression. Such savings on nutrient resources are likely to be significant for enhancing bacterial survival, particularly in harsher and nutrient-deprived environments. This rather low percentage is a striking result, contrasting with much of the current intuition regarding quorate behaviour; it is, however, plausible that under many circumstances such a proportion of up-regulated cells will suffice to achieve the required degree of virulence, say The role of the three mechanisms inferred from the analyses can be summarised as follows.

Background Inhibition (BI). The retarding effect of RsmA on P. aeruginosa is demonstrated in Figure 4A of Pessi et al. [21], whereby the up regulation of QS activity is initiated significantly earlier in a $r s m A^{-}$mutant than in the wild-type. Chugani et al. [6] observed similar results in their experiments comparing wild-type and $q s c R^{-}$strains; however, the repressive action of $q s c R$ is currently uncertain. The analysis in Sections 2.4.2 and 3.2.1 shows that an increasing (decreasing) QSE inhibitor concentration $R_{0}$ lengthens (shortens) the timescale for up-regulation (in agreement with experimental work of Pessi et al. [21]) and reduces (increases) the up-regulated cell fraction. In Sections 2.4.2 and 3.2.1 it was shown that QSE inhibition is a necessary, but not sufficient, mechanism to prevent run away of QSM production; either the incorporation of NF or $\mathrm{SU}$ mechanism, for example, in the model is required to predict the levelling off of QSM concentration observed in our experiments. We note that other mathematical models include a term that describes the "natural" degredation of mRNA-lasI, the effects of which, mathematically speaking, will be indistinguishable from the BI repression process of the current model. The stability of mRNA-lasI is not known, however, and the fact that $P$. aeruginosa has evolved one or more mechanisms to regulate mRNA-lasI (and many other mRNAs) perhaps indicates that their half-life is too long for efficient QS regulation; this in turn suggests that over the timescales of interest (a few hours) natural degradation of mRNA-lasI is negligible.

Negative Feedback (NF). Figure 4A of [6] also shows that the QSM concentration eventually levels off in the batch culture of a $q s c R^{-}$strain, which is presumably due to the activation of the negative feedback process and enhanced production of RsaL, demonstrating that it too has significant effects on QSM production. The modelling suggests that negative feedback has no influence on the important timescales of QS, but only on the final QSM concentration and the up-regulated cell fraction attained. This to be expected as the molecules involved in negative feedback are themselves regulated 
by QSMs. This is in contrast to the viewpoint of de Kievit et al. [7] that the negative feedback function of RsaL in $P$. aeruginosa is to act as a block to up-regulation at low cell densities; the current modelling suggests that the BI or SU mechanisms are far more effective in this regard. It would be interesting to repeat the experiments in Section 2.2 using a $r s a L^{-}$strain of $P$. aeruginosa. Here, assumming $\eta=0$, the model predicts that the QSM concentration will continue to rise in proportion to the bacterial density during the exponential phase of growth, rather than levelling off. If in such an experiment the QSM concentration were observed to level off then there must be some other, currently unknown, repression mechanism regulating QSM production in P. aeruginosa, such as SU mechanism (and not the BI mechanism, for reasons noted above).

Soaking Up of QSMs (SU). The models predict that the QSM soaking-up rate $\eta$ significantly affects both the timescales and large time outcomes. This perhaps is one of the functions of TrlR in QS of A. tumefaciens [4]. It would also be interesting to adapt the experimental procedures described in Section 2.2 to compare QSM output of wild type and $\operatorname{trl} R^{-}$mutants growing in liquid culture. The model predicts that the mutant will, unless there are other regulatory processes operating, up regulate earlier, with QSM levels continually rising throughout the exponential growth phase. It is currently unknown whether this repression mechanism is generic in bacteria, or peculiar to just a few species. Nevertheless, the assumption of QSM degradation is common in other mathematical models and care is therefore needed when assessing the biological relevance of the conclusions drawn from these models' results.

In summary, the modelling suggests that the BI mechanism is necessary to prevent the overproduction of QSMs, but requires further regulatory processes, such as NF and SU mechanisms, to achieve this. Both BI and SU are involved in the speed of up-regulation and NF and SU are important in restricting QSM output and up-regulated cell fraction. Hence the combinations $\mathrm{BI}$ and $\mathrm{NF}$ (as with P. aeruginosa) or BI and SU (perhaps relevent for A. tumefaciens) are sufficient for fine tuning of the timing and extent of up-regulation. The importance and robustness of the BI mechanism, as predicted by the modelling, suggests that it is likely to be a employed by many, if not all, quorum sensing bacteria. To our knowledge, such a process has thus far been identified only in $P$. aeruginosa, in which two regulator proteins (namely RsmA and QscR) are known to be involved. It would therefore be worth seeking equivalent proteins in other bacterial species.

The prediction that the fraction of up-regulated cells may in practice be relatively small is perhaps contrary to the usual dogma that almost the whole population, particularly in cultures, becomes quorate. Experimental work involving QS in growing populations has measured only the global responses and investigations at an individual cell level, to determine proportions of up-regulated cells, have yet to be undertaken. Experiments to ascertain individual responses, say by using a specially constructed reporter strains in which measurements of individual responses can be made, would be very worthwhile, not only to determine whether total or partial up-regulation occurs (which may help validate the model), but also to provide important insights into the behavioural dynamics of a population regulated by QS. Such insights may prove vital in the development of new therapeutic strategies. For example, a drug targeting "up-regulated" cells may not be effective due to most of the population actually being "down-regulated" (as seems to be the case in Figure 4). 
Repression processes in QS are a relatively new area of research and they have thus far been identified in only a few species of bacteria. Such processes may be regulated directly by QS (for example the rsaL system in P. aeruginosa) or regulated by any number of other factors (such as by other independent regulatory processes or by environmental signals). It seems likely that such regulatory processes are widespread, perhaps ubiquitous, in quorum sensing bacteria. It is hoped that the mathematical modelling of this paper offers some useful concrete insights into the potential roles of different repression mechanisms and will stimulate further experimental investigation, a number of specific suggestions having been outlined above.

\section{Acknowledgements}

J.P. Ward gratefully acknowledges support by a Wellcome Trust Training Fellowship in Mathematical Biology (Fellowship ref. 054464/2/98) and the other authors the support of the BBSRC.

\section{References}

[1] K. Anguige, J.R. King, J.P. Ward, and P. Williams. Mathematical modelling of therapies targeted at bacterial quorum sensing. Submitted.

[2] S. Beck von Bodman, D.R. Majerczak, and Coplin D.L. A negative regulator mediates quorum-sensing control of exopolysaccharide productio in Pantoea stewartii subsp. stewartii. Proc. Nat. Acad. Sci. USA, 95:7687-7692, 1998.

[3] S.P. Brown and R.A. Johnstone. Cooperation in the dark: signalling and collective action in quorum-sensing bacteria. Proc. R. Soc. Lond. B, 268:1-5, 2001.

[4] Y. Chai, J. Zhu, and S.C. Winans. TrlR, a defective TraR-like protein of Agrobacterium tumefaciens, blocks TraR function in vitro by forming inactive TrlR:TraR dimers. Mol. Microbial., 40:414-421, 2001.

[5] D.L. Chopp, M.J. Kirisits, M.R. Parsek, and B. Moran. A mathematical model of quorum sensing in a bacterial biofilm. J. Indust. Microbiol. Biotach., 29:339-346, 2002.

[6] S.A. Chugani, M. Whiteley, K.M. Lee, D. D’Argenio, C. Manoil, and E.P. Greenberg. QscR, a modulator of quorum-sensing signal synthesis and virulence in Pseudomonas aeruginosa. Proc. Nat. Acad. Sci. USA, 98:2752-2757, 2001.

[7] T. De Kievit, P.C. Seed, J. Nezezon, L. Passador, and B.H Iglewski. RsaL, a novel repressor of virulence gene expression in Pseudomonas aeruginosa. J. Bacteriol., 181:2175-2184, 1999 .

[8] S.P. Diggle, K. Winzer, A. Lazdunski, P. Williams, and M. Cámara. Advancing the quorum in Pseudomonas aeruginosa: MvaT and the regulation of $\mathrm{N}$-acylhomoserine lactone production and virulence gene expression. J. Bacteriol., 184:2576-2586, 2002.

[9] J.D. Dockery and J.P. Keener. A mathematical model for quorum sensing in Pseudomonas aeruginosa. Bull. Math. Biol., 63:95-116, 2001. 
[10] G. Döring. Pseudomonas aeruginosa infection in cystic fibrosis patients. In M. Campa, M. M. Bendinelli, and H. Friedman, editors, Pseudomonas aeruginosa as an opportunistic pathogen. Plenum Press, New York, 1993.

[11] A. Eberhard, A.L. Burlingame, C. Eberhard, K.H. Kenyon, and N.J. Oppenheimer. Structural identification of the autoinducer of Photobacterium fischeri. Biochem., 20:2444$2449,1981$.

[12] C. Fuqua and E.P. Greenberg. Listening in on bacateria:acyl-homoserine lactone signalling. Molec. Cell Biol., 3:685-695, 2002.

[13] S. Heeb, K. Heurlier, C. Valverde, M. Camera, D. Haas, and Williams P. Posttranscriptional regulation in pseudomonas spp. via the Gac/Rsm regulatory network. In Some editors, editor, Global post-transcriptional regulation. In preparation.

[14] I.A. Holder. Pseudomonas aeruginosa burn infections: pathogenesis and treatment. In M. Campa, M.M. Bendinelli, and H. Friedman, editors, Pseudomonas aeruginosa as an opportunistic pathogen. New York: Plenum Press, 1993.

[15] S. James, P. Nilsson, G. James, S Kjelleberg, and T. Fagerström. Luminescence control in the marine bacterium Vibrio fischeri: an analysis of the dynamics of lux regulation. $J$. Mol. Biol., 296:1127-1137, 2000.

[16] A.J. Koerber, J.R. King, J.P. Ward, P. Williams, J.M. Croft, and R.E. Sockett. A mathematical model of partial-thickness burn-wound infection by Pseudomonas aeruginosa: quorum sensing and the build-up to invasion. Bull. Math. Biol., 64:239-259, 2002.

[17] V. Kõiv and A. Mäe. Quorum sensing controls the synthesis of virulence factors by modulating rsmA gene expression in Erwinia carotovora subsp. carotovora. Mol. Genet. Genom., 265:287-292, 2001.

[18] P. Nilsson, A. Olofsson, M. Fagerlind, T. Fagerström, S. Rice, S. Kjelleberg, and P. Steinberg. Kinetics of the ahl regulatory system in a model biofilm system: how many bacteria constitute a "quorum". J. Mol. Biol., 309:631-640, 2001.

[19] M.R. Parsek and E.P. Greenberg. Acyl-homoserine lactone quorum sensing in Gramnegative bacteria: a signalling mechanism involved in associations with higher organisms. Proc. Nat. Acad. Sci. USA, 97:8789-8793, 2000.

[20] J.P. Pearson, C. van Dalden, and B.H. Iglewski. Active efflux and diffusion are involved in transport of Pseudomonas aeruginosa cell-to-cell signals. J. Bacteriol., 181:1203-1210, 1999.

[21] G. Pessi, F. Williams, Z. Hindle, K. Heurlier, M.T.G. Holden, M. Cámara, D. Haas, and P. Williams. The global posttranscriptional regulator RsmA modulates production of virulence determinants and $N$-acylhomoserine lactones in Pseudomonas aeruginosa. J. Bacteriol., 183:6676-6683, 2001.

[22] K.R. Piper, B. von Vodman, and S.K. Ferrand. Conjugation factor of Agrobacterium tumefaciens regulates Ti plasmid transfer by autoinduction. Nature, 362:448-450, 1993. 
[23] A. Swiderska, A.K. Berndtson, M. Cha, L. Li, G.M.J. Beadoiun III, J. Zhu, and C. Fuqua. Inhibition of the Agrobacterium tumefaciens TraR quorum sensing regulator. J. Biol. Chem., 276:52, 2001.

[24] S. Swift, J.P. Throup, P. Williams, G.P.C. Salmond, and G.S.A.B. Stewart. Quorum sensing: a population density component in the determination of bacterial phenotype. Trends Biochem. Sci., 21:214-219, 1996.

[25] J.P. Ward, J.R. King, A.J. Koerber, J.M. Croft, R.E. Sockett, and P. Williams. Early development and quorum sensing in bacterial biofilms. J. Math. Biol., 47:23-55, 2003.

[26] J.P. Ward, J.R. King, A.J. Koerber, P. Williams, J.M. Croft, and R.E. Sockett. Mathematical modelling of quorum sensing in bacteria. IMA J. Math. Appl. Med. Biol., 18:263$292,2001$.

[27] H. Withers, S. Swift, and P. Williams. Quorum sensing as an integral component of gene regulatory networks in Gram-negative bacteria. Curr. Opin. Microbiol., 4:186-193, 2001.

\section{Appendix A: detailed examination of QSM production}

The purpose of this appendix is to summarise the intracellular biochemistry behind the QSM production rate constants $\kappa_{u}$ and $\kappa_{d}$ in equation (3). The synthesis of QSMs by Gram-negative bacteria involves the transcription of certain genes (belonging to the luxI family of genes) that output an enzyme (QSE) which in turn promotes QSM production by catalysing the reaction of the two QSM precursor molecules, S-adenosylmethionine (SAM) and an appropriate AcylACP (acyl carrier protein) [12]. Figure 2 shows a simplified schematic of the biochemistry of the primary (las) system in $P$. aeruginosa, which can be summarised by the chemical reaction

$$
S+C_{12} \stackrel{\text { LasI }}{\longrightarrow} a+M
$$

where $S, C_{12}, a$ and $M$ are SAM, $\mathrm{C}_{12}$-ACP, 3 -oxo-C12-HSL and the waste product $5^{\prime}$-methylthioadenosine, respectively. We note that both $\mathrm{SAM}$ and $\mathrm{C}_{12}$-ACP are produced independently of quorum sensing. Defining $m$ to be the concentration of mRNA-lasI and $L$ to be the concentration of the QSE LasI, and assuming that the concentrations of SAM and $\mathrm{C}_{12}$-ACP are at fixed constant levels $S_{0}$ and $C_{0}$, respectively, then using the law of mass action we may model QSM production by

$$
\begin{aligned}
\frac{\mathrm{d} m}{\mathrm{~d} t} & =M_{i}-k_{r} R_{0} m \\
\frac{\mathrm{d} L}{\mathrm{~d} t} & =k_{l} m-k_{q} L, \\
\frac{\mathrm{d} a}{\mathrm{~d} t} & =k_{c} S_{0} C_{0} L+k_{b} S_{0} C_{0},
\end{aligned}
$$

where $R_{0}$ is the concentration of the mRNA-lasI degraders, in this case RsmA (but possibly also representing QscR within a single "generic" inhibitor); $M_{i}$ is the mRNA-lasI production rate by down- $(i=d)$ and up- $(i=u)$ regulated cells, $k_{r}$ is the reaction rate of mRNA-lasI breakdown by RsmA, $k_{q}$ is the natural decay rate for LasI (no bacterially produced agent that destroys the enzyme has been identified) and $k_{c}$ and $k_{b}$ are the QSM production rates due 
to the catalysed and background SAM $-\mathrm{C}_{12}$-ACP reactions, respectively. It is assumed that $M_{d} \ll M_{u}$. For the timescales of interest, we can assume that (36) and (37) have attained quasi-steady states, i.e. $m=M_{i} / k_{r} R_{0}$ and $L=k_{l} m / k_{q}=k_{l} M_{i} / k_{q} k_{r} R_{0}$, which on substitution into (38) gives

$$
\frac{\mathrm{d} a}{\mathrm{~d} t}=\frac{k_{l} k_{c} S_{0} C_{0}}{k_{q} k_{r} R_{0}} M_{i}+k_{b} S_{0} C_{0}
$$

from which we can deduce the expressions

$$
\kappa_{d}=\frac{k_{l} k_{c} S_{0} C_{0}}{k_{q} k_{r} R_{0}} M_{d}+k_{b} S_{0} C_{0}, \quad \kappa_{u}=\frac{k_{l} k_{c} S_{0} C_{0}}{k_{q} k_{r} R_{0}} M_{u}+k_{b} S_{0} C_{0},
$$

for the rate constants $\kappa_{d}$ and $\kappa_{u}$, with $\kappa_{d} \ll \kappa_{u}$. The expressions (39) make explicit the role of QSE inhibitor molecules on QSM production, whereby the $\kappa_{i}$ S are approximately inversely proportional (assuming the $k_{b} S_{0} C_{0}$ term to be relatively small) to the QSE inhibitor concentration $R_{0}$.

\section{Appendix B: Asymptotic analysis for exponential phase}

\section{B.1 Introduction}

In this appendix we exploit the fact that $\varepsilon$ is small and seek asympotitic solutions to the system (10) and (11), subject to $n_{u}(0)=0$ and $a(0)=0$, in the limit $\varepsilon \rightarrow 0$. We note that the experimental data suggests there are vast differences between the relative sizes of the parameters, so further simplifications could also be made. However, sufficient progress can be achieved without such modifications; indeed, variability across bacteria species and environmental differences could result in different balances between the parameters, so it is appropriate to seek solutions for rather general cases. The approach is similar to that used in the analysis described in Ward et al. [26] and only brief references are made to those solutions which are eqiuvalent; more detail is given of the new solutions. The analysis of [26] is also generalised here to consider a general initial cell density $n_{T}(0)=N_{0} \ll 1$, rather than $N_{0}=O(\varepsilon)$. As noted in the main text, the most important parameter in governing the evolution of $n_{u}$ and $a$ is

$$
\Theta=\frac{\alpha}{\mu+\eta}+\gamma-\beta-2
$$

In Section B.2 we summarise the analysis for $\Theta=O(1)$, expanding on the work described in [26] to include the QSM repression terms and in Section B.3 we examine more closely the asymptotic behaviour for $|\Theta| \sim 0$, which is the borderline parameter regime. We note that all the analysis to follow applies for the double limit of $\varepsilon \rightarrow 0$ and $N_{0} \rightarrow 0$.

\section{B.2 $\Theta=O(1)$}

In this section we focus on the case where $\Theta=O(1)$ can be of either sign. The important timescales are considered in turn. 


\section{B.2.1 $t=O(1)$}

On this timescale the production of QSMs is preformed chiefly by down-regulated cells and the appropriate rescalings are

$$
t=\bar{t}, \quad n_{u}=N_{0}^{2} \varepsilon \bar{n} \sim N_{0}^{2} \varepsilon \bar{n}_{0}, a=N_{0} \varepsilon \bar{a} \sim N_{0} \varepsilon \bar{a}_{0},
$$

leading to a system and solutions equivalent to (12)-(15) in [26], but with $N_{0}=1$. Of note is the behaviour as $\bar{t} \rightarrow \infty$, which leads to $n_{u}=O\left(\varepsilon N_{0}^{2} \mathrm{e}^{2 t}\right)$ and $a=O\left(\varepsilon N_{0} e^{t}\right)$, so the leading order balance breaks down when $t \sim \ln \left(1 / N_{0}\right)$, when $n_{u}$ becomes comparable to $a$.

B.2.2 $t=\ln \left(1 / N_{0}\right)+O(1)$

On this timescale $n_{u}=O(\varepsilon)$ and $a=O(\varepsilon)$ and, again, the analysis of Section 4.3 of [26] applies. The analysis of this timescale gives rise to the important parameter $\Theta$, which determines whether a substantial proportion of a population can be up regulated. Writing $t=\ln \left(1 / N_{0}\right)+\tilde{t}$, then it can be shown that the leading order solutions for $\tilde{t}=O(1)$ satisfy, when $\Theta>0$,

$$
n_{u} \sim \varepsilon N_{c} \mathrm{e}^{\Theta \tilde{t}} n_{T}, \quad a \sim \varepsilon \frac{N_{c}}{\mu+\eta} \mathrm{e}^{\Theta \tilde{t}},
$$

as $\tilde{t} \rightarrow \infty$ for some constant $N_{c}$, and for $\Theta<0$ we instead have

$$
n_{u} \sim \varepsilon \frac{\alpha}{(\mu+\eta)(-\Theta)} n_{T}, \quad a \sim \varepsilon \frac{\alpha+(\mu+\eta)(-\Theta)}{(\mu+\eta)^{2}(-\Theta)},
$$

as $\tilde{t} \rightarrow \infty$ with $\varepsilon \ll 1$; we note that the equivalent expression for $n_{u}$ of [26] is erroneous and should be as given by (42). We observe from (42) that the expressions are singular as $\Theta \rightarrow 0^{-}$, the leading order balances changing for sufficiently small $\Theta$; this motivates the analysis of Section B.3. We observe from (41) that, for $\Theta>0, n_{u}$ is grows faster than $n_{T}$ and for $\tilde{t}=\ln (1 / \varepsilon) / \Theta+O(1)$ new balances in the system arise. For $\Theta<0$ there are no further timescales of interest and the analysis is complete; we note that $a=O(\varepsilon)$ and there is insufficient QSM accumulation for the negative feedback mechanism to operate at leading order.

B.2.3 $\Theta>0$ and $t=\ln \left(1 / N_{0}\right)+\ln (1 / \varepsilon) / \Theta+O(1)$

The relevant scalings for this timescale are

$$
t=\ln \left(1 / N_{0}\right)+\ln (1 / \varepsilon) / \Theta+t^{\dagger}, \quad n_{u}=\varepsilon^{-1 / \theta} n^{\dagger} \sim \varepsilon^{-1 / \theta} n_{0}^{\dagger}, \quad a=a^{\dagger} \sim a_{0}^{\dagger},
$$

which, by matching with (41) as $t^{\dagger} \rightarrow-\infty$, leads to the leading order system

$$
\begin{aligned}
\frac{\mathrm{d} n_{0}^{\dagger}}{\mathrm{d} t^{\dagger}} & =(\gamma-1-\beta) n_{0}^{\dagger}+\alpha a_{0}^{\dagger}\left(\mathrm{e}^{t^{\dagger}}-n_{0}^{\dagger}\right), \\
n_{0}^{\dagger} & =\frac{(\mu+\eta) a_{0}^{\dagger} \mathrm{e}^{t^{\dagger}}}{\mu a_{0}^{\dagger}+\left(1-g\left(a_{0}^{\dagger}\right)\right)}
\end{aligned}
$$

which combine to give the separable equation

$$
\frac{\mathrm{d} a_{0}^{\dagger}}{\mathrm{d} t^{\dagger}}=\frac{1-g\left(a_{0}^{\dagger}\right)+\mu a_{0}^{\dagger}}{(\mu+\eta)\left(1-g\left(a_{0}^{\dagger}\right)+a_{0}^{\dagger} g^{\prime}\left(a_{0}^{\dagger}\right)\right)} P\left(a_{0}^{\dagger}\right) a_{0}^{\dagger},
$$


where $g^{\prime}(a)=\mathrm{d} g / \mathrm{d} a$ and

$$
P(a)=(\mu+\eta) \Theta-\alpha \eta a-\alpha g(a) .
$$

Since $g\left(a_{0}^{\dagger}\right) \leq g_{\infty} \leq 1$ and $g\left(a_{0}^{\dagger}\right) \rightarrow 0$ as $t^{\dagger} \rightarrow-\infty$ the right-hand side of (45) is positive, hence $a_{0}^{\dagger}$ is monotonic increasing. Since $P(0)=(\mu+\eta) \Theta>0$ and $P^{\prime}(a)=-\alpha\left(\eta+g^{\prime}(a)\right)<0$, the large-time behaviour of (45) is dependent on whether there exists a steady-state $a_{0}^{\dagger *}>0$ such that $P\left(a_{0}^{\dagger^{*}}\right)=0$. There are two cases to consider, namely $\eta>0$ (where $a_{0}^{\dagger^{*}}>0$ always exist) and $\eta=0$ (where a solution $a_{0}^{\dagger *}>0$ exists only if $\mu \Theta / \alpha<g_{\infty}$ ).

$\eta>0$. Since $P(a) \rightarrow-\infty$ as $a \rightarrow \infty$ there exists a unique $a_{0}^{\dagger^{*}}>0$ such that $P\left(a_{0}^{\dagger^{*}}\right)=0$, with

$$
a \sim a_{0}^{\dagger *}=P^{-1}(0), \quad n_{u} \sim \frac{(\mu+\eta) a_{0}^{\dagger *}}{\mu a_{0}^{\dagger *}+1-g\left(a_{0}^{\dagger *}\right)} n_{T},
$$

as $t^{\dagger} \rightarrow \infty$. We note in the absence of the NF mechanism, i.e. $g(a) \equiv 0$, then $a_{0}^{\dagger *}=$ $(\mu+\eta) \Theta / \alpha \eta$.

$\eta=0$. In this case $P(a)$ reduces to $P(a)=\mu \Theta-\alpha g(a)$ and the large-time outcomes are dependent on whether (i) $g_{\infty}>\mu \Theta / \alpha$, (ii) $g_{\infty}=\mu \Theta / \alpha$ or (iii) $g_{\infty}<\mu \Theta / \alpha$.

(i) $g_{\infty}>\mu \Theta / \alpha$. In this case $a_{0}^{\dagger}$ tends to a steady-state $a_{0}^{\dagger *}=g^{-1}(\mu \Theta / \alpha)$, as $t^{\dagger} \rightarrow \infty$ and

$$
a \sim a_{0}^{\dagger^{*}}, \quad n_{u} \sim \frac{\mu a_{0}^{\dagger^{*}}}{\mu a_{0}^{\dagger^{*}}+1-g\left(a_{0}^{\dagger^{*}}\right)} n_{T},
$$

as $t^{\dagger} \rightarrow \infty$. The analysis for this case is then complete.

(ii) $g_{\infty}=\mu \Theta / \alpha$. In this case, the right-hand side of (45) vanishes only when $a_{0}^{\dagger} \rightarrow \infty$ and for large $a_{0}^{\dagger}$ we have

$$
\frac{\mathrm{d} a_{0}^{\dagger}}{\mathrm{d} t^{\dagger}} \sim \frac{\alpha}{\Theta} \bar{g}\left(a_{0}^{\dagger}\right) a_{0}^{\dagger}
$$

as $t^{\dagger} \rightarrow \infty$, where $\bar{g}(a)=\mu \Theta / \alpha-g(a)$, so that $\bar{g}(\infty)=0$ and $\bar{g}^{\prime}(a)<0$. The evolution of $a_{0}^{\dagger}$ in large time depends on the behaviour of $\bar{g}\left(a_{0}^{\dagger}\right)$ as $a_{0}^{\dagger} \rightarrow \infty$. However, since $\bar{g}(a)$ vanishes as $a \rightarrow \infty$ growth of $a_{0}^{\dagger}$ is sub-exponential; consequently, there are no further timescales of interest to consider. Using the original variables, the outcomes for two likely candidates for $\bar{g}(a)$ as $a \rightarrow \infty$ are

$$
n_{u} \sim n_{T}, \quad a \sim\left\{\begin{aligned}
\left(\frac{c \alpha(m+1)}{\Theta}\right)^{\frac{1}{m+1}} t^{\dagger / m+1} & \bar{g}(a) & \sim \frac{c}{a^{(m+1)}}, \\
\frac{1}{m} \ln \left(t^{\dagger}\right) & \bar{g}(a) & \sim c \mathrm{e}^{-m a},
\end{aligned}\right.
$$

as $t^{\dagger} \rightarrow \infty$, where $m$ and $c$ are positive constants.

(iii) $g_{\infty}<\mu \Theta / \alpha$. Again equation (45) has no steady-states and $a_{0}^{\dagger}$ will continue to grow. Assuming, for simplicity, that $a g^{\prime}(a) \rightarrow 0$ as $a \rightarrow \infty$, as would be likely for relevant forms of $g(a)$, we have

$$
\frac{\mathrm{d} a_{0}^{\dagger}}{\mathrm{d} t^{\dagger}} \sim \frac{\mu \Theta-\alpha g_{\infty}}{\mu\left(1-g_{\infty}\right)}\left(\left(1-g_{\infty}\right) a_{0}^{\dagger}+\mu a_{0}^{\dagger^{2}}\right)
$$


as $t^{\dagger} \rightarrow \infty$, a separable equation that solves to give

$$
a_{0}^{\dagger} \sim \frac{\left(1-g_{\infty}\right) \mathrm{e}^{\mu Z t^{\dagger}}}{\left(1-g_{\infty}\right) A_{0}-\mu \mathrm{e}^{\mu Z t^{\dagger}}}
$$

where $A_{0}$ is a positive constant of integration and $Z=\left(\mu \Theta-\alpha g_{\infty}\right) / \mu\left(1-g_{\infty}\right)$. We note from (44) that

$$
n_{0}^{\dagger} \sim N_{0} \mathrm{e}^{t^{\dagger}}
$$

as $t^{\dagger} \rightarrow \infty$. Here the expansions fail when the denominator of (50) becomes zero, corresponding to $t^{\dagger} \sim t_{c}^{\dagger}(0)=\ln \left(\left(1-g_{\infty}\right) A_{0} / \mu\right) / \mu Z$, leading to blow up of the solution $a_{0}^{\dagger}$. The analysis about the transition period corresponding to the near blow-up region is a straightforward extension to the analysis described in Sections 4.4.2 and 4.4.3 in [26] and we will omit the details. However, following this transition period a further rescaling of the form

$$
t=\ln \left(1 / N_{0}\right)+\ln (1 / \varepsilon) / \Theta+t^{\ddagger}, \quad n_{u}=\varepsilon^{-1 / \Theta} n^{\ddagger}, \quad a=\varepsilon^{-1 / \Theta} a^{\ddagger},
$$

eventually leads to the large time solutions

$$
n_{u} \sim n_{T}, \quad a \sim \frac{\mu \Theta-\alpha g_{\infty}}{\alpha(1+\lambda)} n_{T},
$$

as $t^{\ddagger} \rightarrow \infty$. We note that $a$ grows exponentially, in contrast to the $g_{\infty}>\mu \Theta / \alpha$ case, and that the solutions of [26] can be derived by setting $g_{\infty}=0$ in (53).

The analysis demonstrates that in order to get saturation of QSM concentration during exponential population growth with small $\varepsilon$, we need either $\eta>0$ or $g_{\infty}<\mu \Theta / \alpha$, as $\varepsilon \rightarrow 0$.

\section{B.3 $|\Theta| \ll 1$}

In this section we examine the evolution of $n_{u}$ and $a$ in the vicinity of $\Theta=0$. It turns out that the evolution of $a$ and $n_{u}$ in this limit is governed by the behaviour of $g(a)$ as $a \rightarrow 0$. We shall consider a fairly general case in which

$$
g(a) \sim B a^{m},
$$

as $a \rightarrow 0$ where $B$ and $m$ are both positive constants. Rather than consider the case of $\Theta=0$, as we did in [26], it is more instructive to seek solutions for small $\Theta$ and, given (54), the appropriate scaling for the relevant distinguished limit is

$$
\Theta= \begin{cases}\varepsilon^{\frac{m}{m+1}} \theta & m<1, \\ \varepsilon^{\frac{1}{2}} \theta & m \geq 1,\end{cases}
$$

the latter case implying no negative feedback. The analysis below starts from the $t=$ $\ln \left(1 / N_{0}\right)+O(1)$ timescale, where the solution types diverge depending on the sign of $\Theta$. 
B.3.1 $t=\ln \left(1 / N_{0}\right)+O(1)$

The appropriate rescalings are

$$
t=\ln \left(1 / N_{0}\right)+\tilde{t}, n_{u} \sim \varepsilon \tilde{n}_{0}+\varepsilon^{\delta+1} \tilde{n}_{1}, a \sim \varepsilon \tilde{a}_{0}+\varepsilon^{\delta+1} \tilde{a}_{1},
$$

where $\delta=m /(m+1)$ and $\delta=1 / 2$ for the $m<1$ and $m \geq 1$ cases, respectively, which, for brevity combining the leading- and second-order expressions, leads to

$$
\begin{aligned}
\frac{\mathrm{d} \tilde{N}}{\mathrm{~d} \tilde{t}} & =\tilde{N}+\alpha \tilde{A} \mathrm{e}^{\tilde{t}}-\frac{\alpha}{\mu+\eta} \tilde{N}+\varepsilon^{\delta} \theta \tilde{n}_{1}, \\
\frac{\mathrm{d} \tilde{A}}{\mathrm{~d} \tilde{t}} & =\tilde{N}-(\mu+\eta) \tilde{A} \mathrm{e}^{\tilde{t}}-\lambda \tilde{A}+\mathrm{e}^{\tilde{t}}
\end{aligned}
$$

where $\tilde{N}=\tilde{n}_{0}+\varepsilon^{\delta} \tilde{n}_{1}$ and $\tilde{A}=\tilde{a}_{0}+\varepsilon^{\delta} \tilde{a}_{1}$. Of most interest is the large time behaviour, namely

$$
\begin{aligned}
n_{u} & \sim \varepsilon \tilde{N} \sim \varepsilon\left(\frac{\alpha}{\mu+\eta} \tilde{t}+\varepsilon^{\delta} \frac{1}{2} \frac{\alpha \theta}{\mu+\eta} \tilde{t}^{2}\right) n_{T}, \\
a & \sim \varepsilon \tilde{A} \sim \varepsilon \frac{\alpha}{(\mu+\eta)^{2}} \tilde{t}+\varepsilon^{\delta+1} \frac{1}{2} \frac{\alpha \theta}{(\mu+\eta)^{2}} \tilde{t}^{2},
\end{aligned}
$$

as $\tilde{t} \rightarrow \infty$. We observe that the correction terms are of the same order as the leading terms when $\tilde{t}=O\left(\varepsilon^{-\delta}\right)$, marking the onset of a new balance in the system. We note that for $\Theta=0$ (i.e. $\theta=0$ ) and $\eta=0$, the analysis of [26] is recovered and the expansions breakdown when $\tilde{t}=O(1 / \varepsilon)$.

\section{B.3.2 $t=\ln \left(1 / N_{0}\right)+O\left(\varepsilon^{-\delta}\right)$}

The appropriate rescalings are

$$
t=\ln \left(1 / N_{0}\right)+\varepsilon^{-\delta} t^{\dagger}, \quad n_{u} \sim \varepsilon^{1-\delta} \mathrm{e}^{\varepsilon^{-\delta} t^{\dagger}} n_{0}^{\dagger}, \quad a \sim \varepsilon^{1-\delta} a_{0}^{\dagger},
$$

noting that $1-\delta>0$ in both cases, which, to leading order, yields

$$
n_{0}^{\dagger}=(\mu+\eta) a_{0}^{\dagger},
$$

and

$$
\frac{\mathrm{d} a_{0}^{\dagger}}{\mathrm{d} t^{\dagger}}= \begin{cases}\frac{\alpha}{(\mu+\eta)^{2}}+\theta a_{0}^{\dagger}-\frac{\alpha B}{\mu+\eta} a_{0}^{\dagger m+1} & m<1, \\ \frac{\alpha}{(\mu+\eta)^{2}}+\theta a_{0}^{\dagger}-\frac{\alpha(B+\eta)}{\mu+\eta} a_{0}^{\dagger^{2}} & m=1, \\ \frac{\alpha}{(\mu+\eta)^{2}}+\theta a_{0}^{\dagger}-\frac{\alpha \eta}{\mu+\eta} a_{0}^{\dagger^{2}} & m>1,\end{cases}
$$

subject to $a_{0}^{\dagger}(0)=0$ (by matching). For $m<1$ the large time behaviour is

$$
a \sim \varepsilon^{\frac{1}{m+1}} a_{0}^{\dagger *}, \quad n_{u} \sim \varepsilon^{\frac{1}{m+1}}(\mu+\eta) a^{*} n_{T},
$$

as $t^{\dagger} \rightarrow \infty$, where the positive constant $a_{0}^{\dagger^{*}}$ satisfies $p\left(a_{0}^{\dagger^{*}}\right)=0$, where

$$
p(a)=a^{m+1}-\frac{\theta(\mu+\eta)}{\alpha B} a-\frac{1}{(\mu+\eta) B} ;
$$


it is straightforward to show that there is only one such solution for $a^{*}$ satisfying $p\left(a^{*}\right)=0$. For $m \geq 1$ we have

$$
a \sim \varepsilon^{\frac{1}{2}} a_{0}^{\dagger *} n_{u} \sim \varepsilon^{\frac{1}{2}}(\mu+\eta) a_{0}^{\dagger *} n_{T}
$$

as $t^{\dagger} \rightarrow \infty$ and $\varepsilon \rightarrow 0$, where

$$
a_{0}^{\dagger^{*}}= \begin{cases}\frac{(\eta+\mu)^{2} \theta+\sqrt{\theta^{2}(\mu+\eta)^{4}+4 \alpha^{2} \eta(\mu+\eta)}}{2 \alpha \eta(\mu+\eta)} & m>1 \\ \frac{(\eta+\mu)^{2} \theta+\sqrt{\theta^{2}(\mu+\eta)^{4}+4 \alpha^{2} \eta(B+\mu+\eta)}}{2 \alpha \eta(B+\mu+\eta)} & m=1 .\end{cases}
$$

We note that (60)-(63) as $\theta \rightarrow+\infty$ and $\theta \rightarrow-\infty$ matches with (47) and (48) as $\Theta \rightarrow 0^{+}$ and with (42) as $\Theta \rightarrow 0^{-}$, respectively.

\section{Appendix C: Asymptotic analysis for fixed depth biofilm}

\section{C.1 Introduction}

In this appendix we study the system (27) and (28) in the limit $\varepsilon \rightarrow 0$. The purpose of the analysis is to investigate the important timescales and what, if any, are the effects of repression processes on the evolution of $N_{u}$ and $A$. An important parameter drawn out by the analysis below is

$$
\Psi_{b}=\alpha-\beta(\eta+\lambda+Q+\mu)
$$

whereby the sign of $\Psi_{b}$ governs whether substantial up regulation can occur. Most of the details of the analysis are covered in the appendix of Ward et al. [25] and are only summarised here.

\section{C.2 $\Psi_{b}=O(1)$}

In this section we seek solutions for the case $\Psi_{b}=O(1)$, where $\Psi_{b}$ could be of either sign.

\section{C.2.1 $t=O(1)$}

On this timescale the down-regulated population governs the QSM production and $N_{u}=O(\varepsilon)$ and $A=O(\varepsilon)$ are both very small. The feedback term has no leading-order effect on this timescale and leads to the solutions given in [25], whereby as $t \rightarrow \infty$ we have when $\Psi_{b}<0$

$$
N_{u} \sim \varepsilon \frac{\alpha}{\left(-\Psi_{b}\right)}, \quad A \sim \varepsilon \frac{\beta}{\left(-\Psi_{b}\right)}
$$

i.e. both tend to constant levels of size $O(\varepsilon)$, and when $\Psi_{b}>0$

$$
N_{u} \sim \varepsilon \frac{\alpha\left(-\omega_{-}\right)}{\Psi_{b}\left(\omega_{+}-\omega_{-}\right)} \mathrm{e}^{\omega_{+} t}, \quad A \sim \varepsilon \frac{\Psi_{b}-\beta \omega_{-}}{\Psi_{b}\left(\omega_{+}-\omega_{-}\right)} \mathrm{e}^{\omega_{+} t},
$$

where

$$
\omega_{ \pm}=-\frac{\beta+\mu+\eta+\lambda+Q}{2} \pm \frac{1}{2} \sqrt{(\beta+\mu+\eta+\lambda+Q)^{2}+4 \Psi_{b}}
$$


we note that $\omega_{+}>0$ and $\omega_{-}<0$ when $\Psi_{b}>0$ and that for $\Psi_{b}>0$ the balance at leading order breaks down when $t=\ln (1 / \varepsilon) / \omega_{+}+O(1)$, whilst for $\Psi_{b}<0$ the analysis at leading order is complete. The apparent incompatibility of solutions as $\Psi_{b} \rightarrow 0^{-}$and as $\Psi_{b} \rightarrow 0^{+}$is explained by the analysis in Section C.3.

C.2.2 $t=\ln (1 / \varepsilon) / \omega_{+}+O(1)$

As with the no-feedback case, the appropriate rescalings for this timescale, with $\Psi_{b}>0$, are given by

$$
t=\ln (1 / \varepsilon) / \omega_{+}+t^{\dagger}, \quad N_{u}=N^{\dagger} \sim N_{0}^{\dagger}, \quad A=A^{\dagger} \sim A_{0}^{\dagger},
$$

which yields at leading order

$$
\begin{aligned}
\frac{\mathrm{d} N_{0} \dagger}{\mathrm{d} t^{\dagger}} & =\alpha A_{0}^{\dagger}\left(1-N_{0}^{\dagger}\right)-\beta N_{0}^{\dagger}, \\
\frac{\mathrm{d} A_{0}^{\dagger}}{\mathrm{d} t^{\dagger}} & =N_{0}^{\dagger}\left(1-G\left(A_{0}^{\dagger}\right)\right)-\mu A_{0}^{\dagger}\left(1-N_{0}^{\dagger}\right)-(\eta+\lambda+Q) A_{0}^{\dagger}
\end{aligned}
$$

as $\varepsilon \rightarrow 0$, where matching with (66) gives

$$
N_{0} \sim \frac{\alpha\left(-\omega_{-}\right)}{\Psi_{b}\left(\omega_{+}-\omega_{-}\right)} \mathrm{e}^{\omega_{+} t^{\dagger}}, \quad A_{0} \sim \frac{\Psi_{b}-\beta \omega_{-}}{\Psi_{b}\left(\omega_{+}-\omega_{-}\right)} \mathrm{e}^{\omega_{+} t^{\dagger}}
$$

as $t^{\dagger} \rightarrow-\infty$. Solutions for (68)-(70) cannot be determined in closed form; however, as $t^{\dagger} \rightarrow \infty$ the solutions $N_{u}$ and $A$ tend exponentially towards a steady state, say $N_{u} \sim N_{u}^{*}$ and $A \sim A^{*}$, satisfying

$$
W\left(A^{*}\right)=0, \quad N_{u}^{*}=\frac{\alpha A^{*}}{\alpha A^{*}+\beta},
$$

where

$$
W(A)=G(A)+(\eta+\lambda+Q) A-\frac{\Psi_{b}}{\alpha},
$$

We note that, since $W(0)=-\Psi_{b} / \alpha<0, W(A) \rightarrow \infty$ as $A \rightarrow \infty$ and $W^{\prime}(A)>0$, there is only one solution with $A^{*}>0$; hence the steady-state is unique. There are no further timescales of interest.

\section{C.3 $\left|\Psi_{b}\right| \ll 1$}

In this section we investigate the behaviour of solutions in the vicinity of the bifurcation between negligible and substantial up regulation of a population within a developing biofilm. As with the analysis described in Appendix B.3, QSM levels remains low and we assume that $G(A) \sim B A^{m}$ as $A \rightarrow 0$; the appropriate rescalings for $\Psi$ are then

$$
\Psi_{b}= \begin{cases}\varepsilon^{\frac{1}{2}} \psi & m \geq 1, \\ \varepsilon^{\frac{m}{m+1}} \psi & m<1 .\end{cases}
$$

We note the analysis below generalises that of [25] where only the case $\Psi_{b}=0$ is considered. 
C.3.1 $t=O(1)$

The analysis of the [25] is modified using the rescaling (73) to give

$$
A \sim \varepsilon \frac{\beta}{\beta+\mu+\eta+\lambda+Q} t+\varepsilon^{\sigma+1} \frac{1}{2} \frac{\beta \psi}{(\beta+\mu+\eta+\lambda+Q)^{2}} t^{2}, \quad N_{u} \sim \frac{\alpha}{\beta} A,
$$

as $t \rightarrow \infty$, where $\sigma=1 / 2$ and $\sigma=m /(m+1)$ for $m \geq 1$ and $m<1$, respectively. We note that the leading and correction terms become of the same order (i.e. the expansion disorders) when $t=O\left(\varepsilon^{1 / 2}\right)$ for $m \geq 1$ and $t=O\left(\varepsilon^{m / m+1}\right)$ for $m<1$, marking the onset of a new balance in the limit $\varepsilon \rightarrow 0$.

\section{C.3.2 Large time}

We consider the cases of $m \geq 1$ and $m<1$ separately.

$m \geq 1$. The appropriate rescalings are

$$
t=t^{\dagger} / \varepsilon^{1 / 2}, \quad N_{u}=\varepsilon^{1 / 2} N^{\dagger} \sim \varepsilon^{1 / 2} N_{0}^{\dagger}, \quad A=\varepsilon^{1 / 2} A^{\dagger} \sim \varepsilon^{1 / 2} A_{0}^{\dagger},
$$

from which we can deduce

$$
\begin{aligned}
N_{0}^{\dagger} & =\frac{\alpha}{\beta} A_{0}^{\dagger}, \\
\frac{\mathrm{d} A_{0}^{\dagger}}{\mathrm{d} t} & =\frac{1}{\beta+\mu+\eta+\lambda+Q}\left(\beta+\psi A_{0}^{\dagger}-\alpha A_{0}^{\dagger^{2}}(\eta+\lambda+Q)-\varepsilon^{m-1} B A_{0}^{\dagger}{ }^{m+1}\right),
\end{aligned}
$$

as $\varepsilon \rightarrow 0$, subject to $A_{0}^{\dagger}(0)=0$ (by matching with (74)). We note that the feedback response term in equation (76) (i.e. he $B$ term) does not of course appear in these leading-order euations when $m>1$. For both cases $m=1$ and $m>1$, equation (76) can be integrated explicitly, and as $t \rightarrow \infty$ we have the steady-states

$$
A \sim \sqrt{\varepsilon} \begin{cases}\frac{1}{2} \frac{\psi+\sqrt{\psi^{2}+4 \alpha \beta(\eta+\lambda+Q)}}{\alpha(\eta+\lambda+Q)} & m>1, \\ \frac{1}{2} \frac{\psi+\sqrt{\psi^{2}+4 \alpha \beta(B+\eta+\lambda+Q)}}{\alpha(B+\eta+\lambda+Q)} & m=1,\end{cases}
$$

and $N_{u} \sim \alpha A / \beta$ from (75).

$m<1$. The appropriate rescalings are

$$
t=\varepsilon^{-\frac{m}{m+1}} t^{\dagger}, \quad N_{u}=\varepsilon^{\frac{1}{m+1}} N^{\dagger} \sim \varepsilon^{\frac{1}{m+1}} N_{0}^{\dagger}, \quad A=\varepsilon^{\frac{1}{m+1}} A^{\dagger} \sim \varepsilon^{\frac{1}{m+1}} A_{0}^{\dagger},
$$

leading to $(75)$ and

$$
\frac{\mathrm{d} A_{0}^{\dagger}}{\mathrm{d} t}=\frac{1}{\beta+\mu+\eta+\lambda+Q}\left(\beta+\psi A_{0}^{\dagger}-\alpha B A_{0}^{\dagger m+1}\right)
$$

subject to $A_{0}^{\dagger}(0)=0$. Hence $A$ evolves to a steady state $A \sim \varepsilon^{1 /(m+1)} A^{*}$ as $t \rightarrow \infty$, with $A^{*}$ satisfying $q\left(A^{*}\right)=0$, where

$$
q(A)=A^{m+1}-\frac{\psi}{\alpha B} A-\frac{\beta}{\alpha B} ;
$$

it is straightforward to show that there is only one positive solution $A^{*}$ satisfying $q\left(A^{*}\right)=$ 0 . From (75) we have $N_{u} \sim \alpha A^{*} / \beta$ as $t \rightarrow \infty$.

We note that these steady-states can be shown to match as $\psi \rightarrow+\infty$ with (71) for $\Psi_{b} \rightarrow 0^{+}$ and as $\psi \rightarrow-\infty$ with (65) for $\Psi_{b} \rightarrow 0^{-}$. 


\section{List of Figures}

1 Schematic of the feedback process of the primary QS system in Pseudomonas aeruginosa. The italics within the large arrows represent the genes producing LasR (the QSP), 3-oxo-C12-HSL (the QSM) and the negative feedback protein RsaL. Vfr and GacA are two external regulators known to be involved in the production of LasR, and RsmA, while QscR is believed to be external repressors of QSM production. The solid curves (labelled with a $(+)$ ) and dashed curves (labelled with a $(-))$ are the positive and negative influences, respectively, on the system. . . . . . . . . . . . . . . . .

2 Schematic of the transcription of the lasI gene in the production of QSM 3-oxoC12-HSL. The gene encodes the QSE LasI, which catalyses the reaction between SAM (S-adenosylmethionine) and $\mathrm{C}_{12}$-ACP to produce the QSM and the waste product MTA (5'-methylthioadenosine). RsmA is a global translational regulator protein which destroys messenger RNA including that transcripted from the lasI gene. . . . . . . . . . . . . . . . . . . .

3 Plot of QSM concentration against time, comparing model solutions using bestfit parameters for BI-NF $\left(g_{\infty}>0, \eta=0\right.$, solid curve), BI-SU $\left(g_{\infty}=0, \eta>0\right.$, dashed curve) and BI only $\left(g_{\infty}=0, \eta=0\right.$, dotted curve) cases with experimental data for QSM production in LB (indicated by the $\bullet$ s). The BI only case cannot predict saturation of QSM concentration and the curve shown is the best fit with data up $t=4$ hours. We note that the quantities involved are dimensional. . . . . . . . . . . . . . . .

4 Predicted evolution of the up-regulated cell fraction using the best-fit parameters listed in Table 1 for BI-NF (solid curve), BI-SU (dashed curve) and BI only (dotted curve) cases. . . . . . . . . . . . . . . .

5 Plot of the evolution of the population density (solid curves) and drug concentration (dashed curves) in response to initial drug dosages of $w_{0}=16$ and $w_{0}=17$, with $\delta=10$. The other dimensionless parameter values are determined from the data given in the first column in Table $1 . \ldots . . . . . . .$.

6 Plot of the bifurcation curves in $\delta-w_{0}$ space for various values of $g_{\infty}$. Parameter regimes above each curve result ultimately result in an exponential decline of the population, below each curve the population will ultimately grow exponentially. The other dimensionless parameter values are determined from the data given in the first column in Table $1 . \ldots \ldots \ldots$. . . . . . . . . . . . 34 


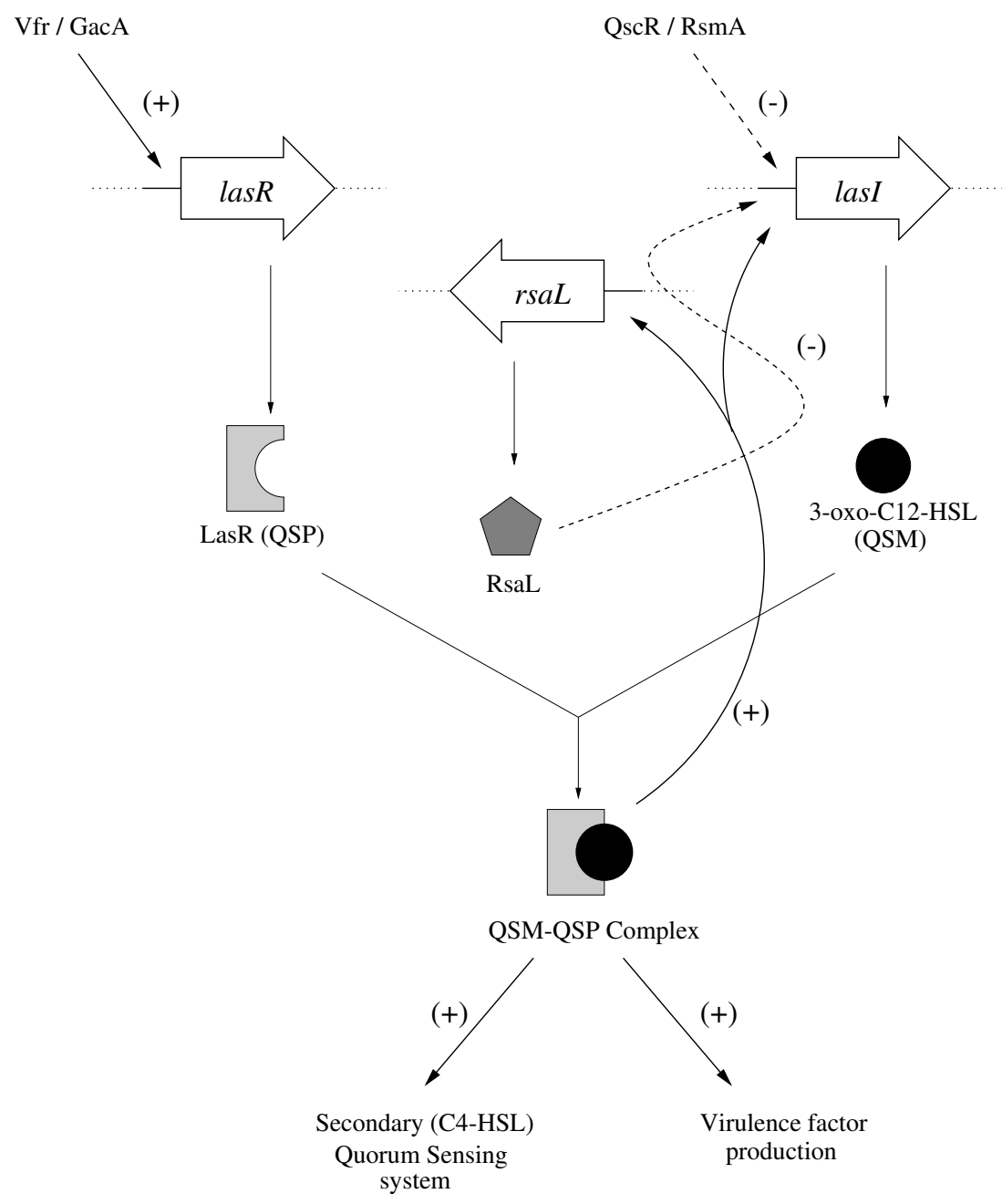

Figure 1: Schematic of the feedback process of the primary QS system in Pseudomonas aeruginosa. The italics within the large arrows represent the genes producing LasR (the QSP), 3-oxo-C12-HSL (the QSM) and the negative feedback protein RsaL. Vfr and GacA are two external regulators known to be involved in the production of LasR, and RsmA, while QscR is believed to be external repressors of QSM production. The solid curves (labelled with a $(+)$ ) and dashed curves (labelled with a (-)) are the positive and negative influences, respectively, on the system. 


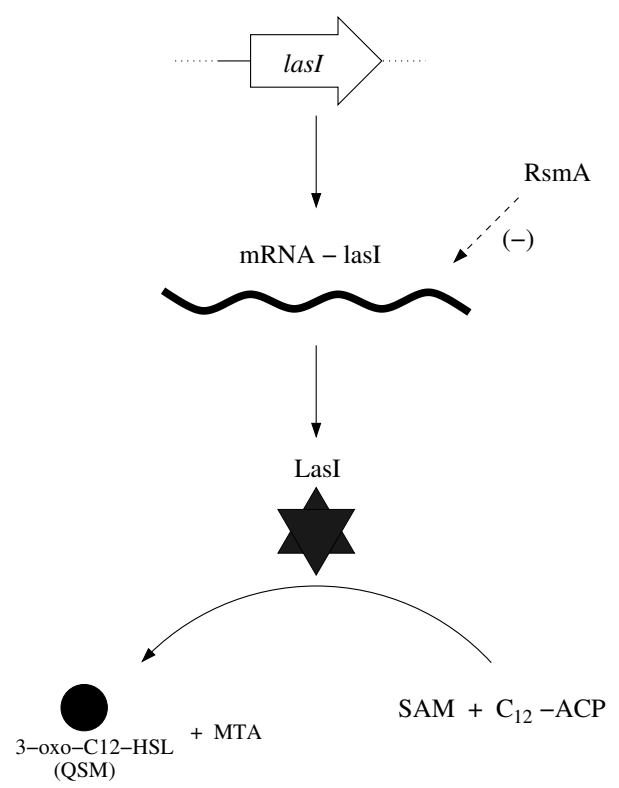

Figure 2: Schematic of the transcription of the lasI gene in the production of QSM 3-oxoC12-HSL. The gene encodes the QSE LasI, which catalyses the reaction between SAM (Sadenosylmethionine) and $\mathrm{C}_{12}$ - $\mathrm{ACP}$ to produce the QSM and the waste product MTA (5'methylthioadenosine). RsmA is a global translational regulator protein which destroys messenger RNA including that transcripted from the lasI gene.

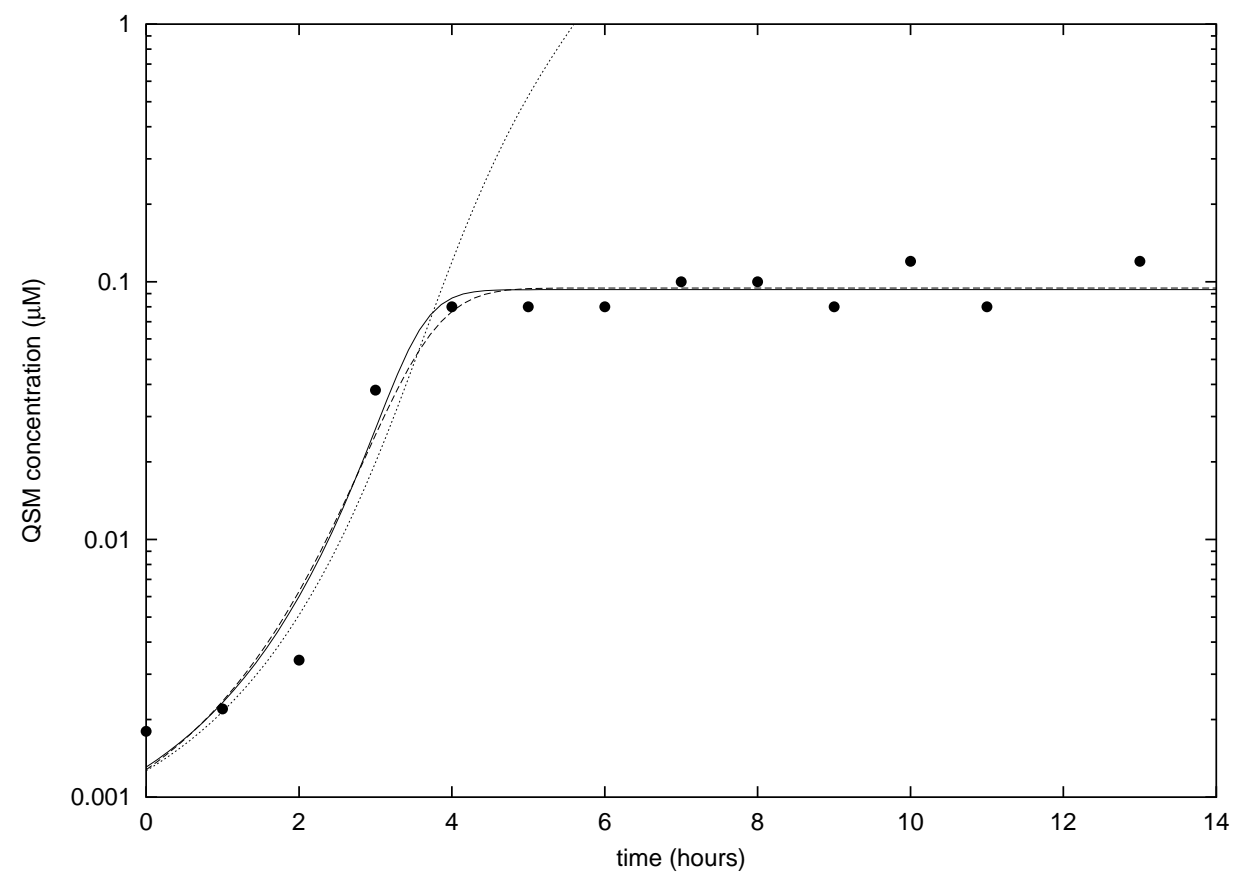

Figure 3: Plot of QSM concentration against time, comparing model solutions using best-fit parameters for BI-NF $\left(g_{\infty}>0, \eta=0\right.$, solid curve), BI-SU ( $g_{\infty}=0, \eta>0$, dashed curve) and BI only $\left(g_{\infty}=0, \eta=0\right.$, dotted curve) cases with experimental data for QSM production in LB (indicated by the $\bullet$ s). The BI only case cannot predict saturation of QSM concentration and the curve shown is the best fit with data up $t=4$ hours. We note that the quantities involved are dimensional. 


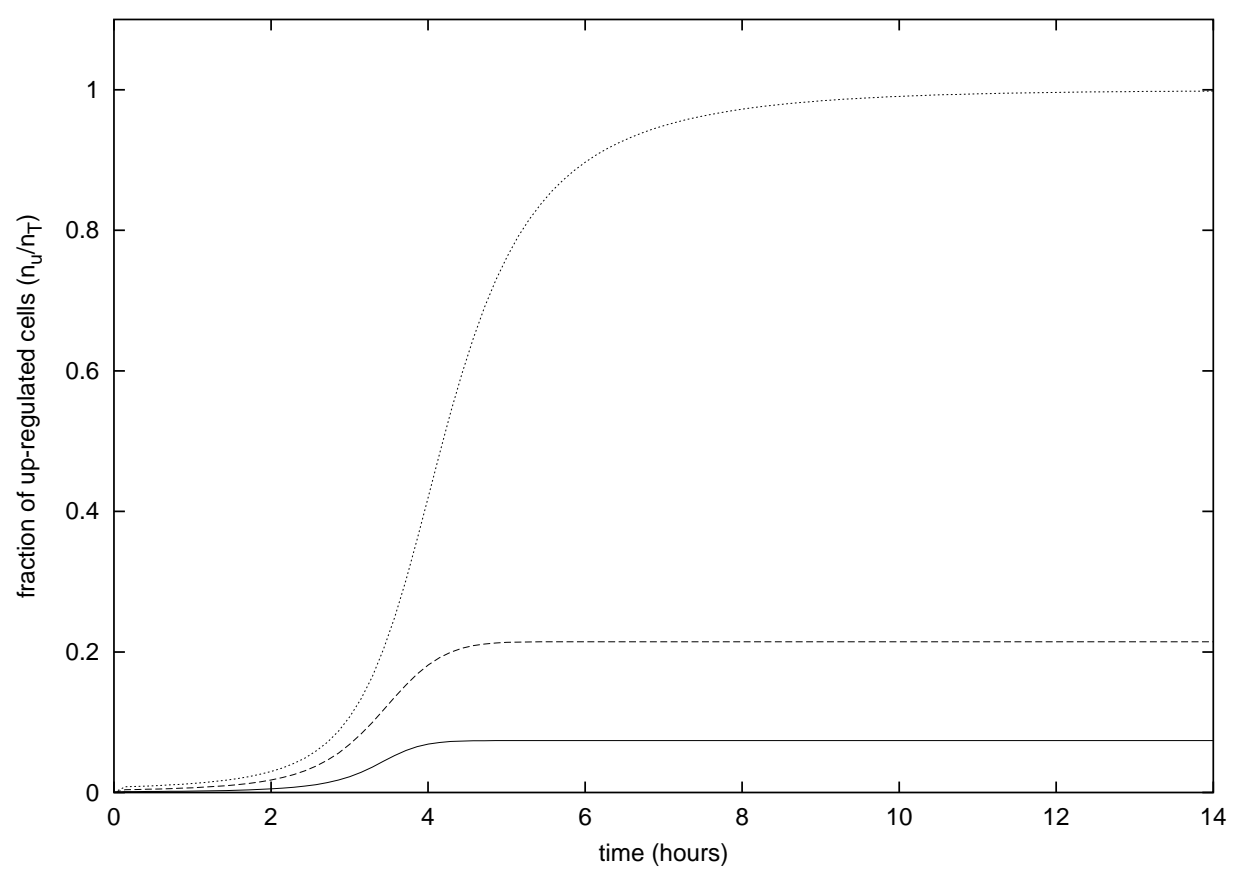

Figure 4: Predicted evolution of the up-regulated cell fraction using the best-fit parameters listed in Table 1 for BI-NF (solid curve), BI-SU (dashed curve) and BI only (dotted curve) cases.

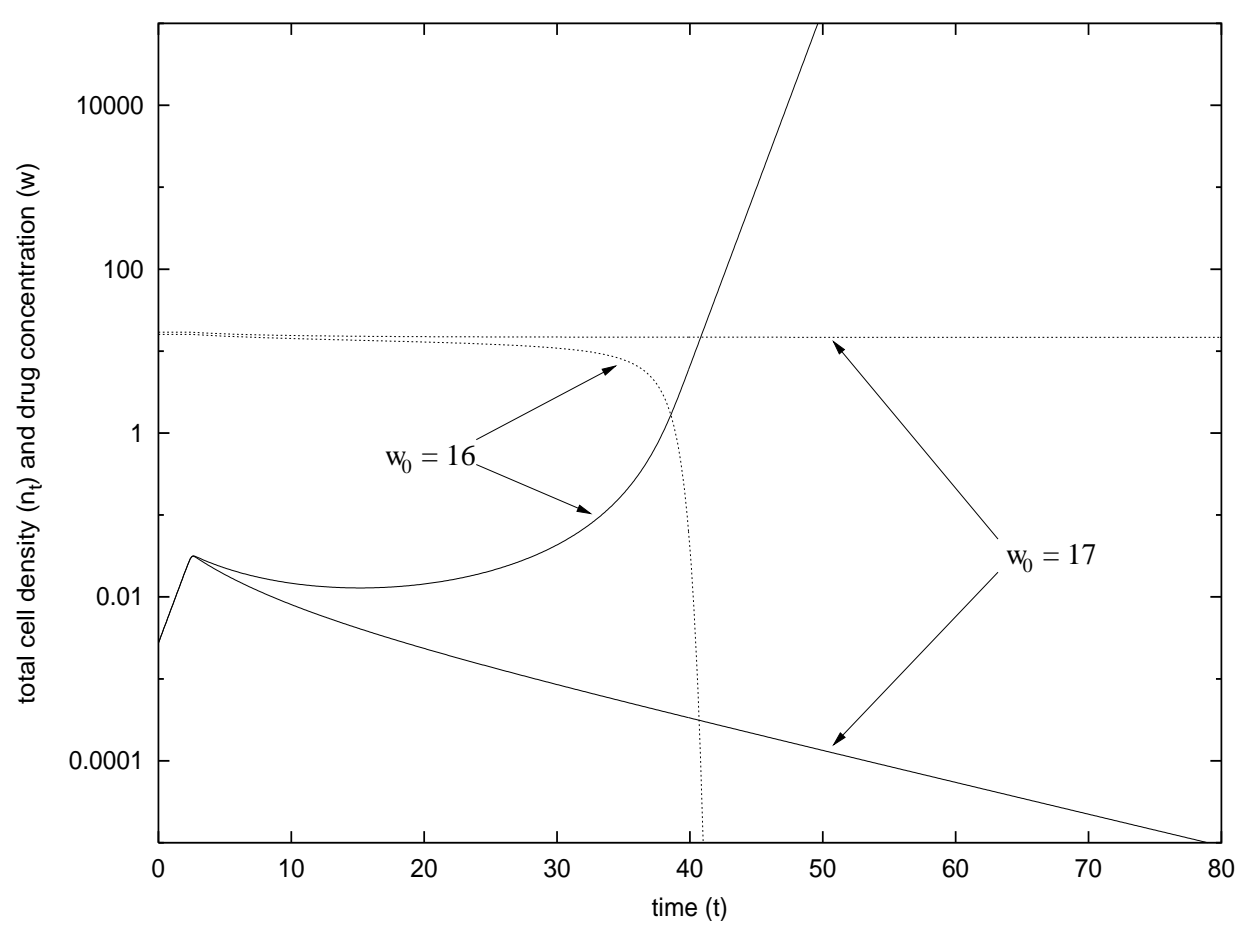

Figure 5: Plot of the evolution of the population density (solid curves) and drug concentration (dashed curves) in response to initial drug dosages of $w_{0}=16$ and $w_{0}=17$, with $\delta=10$. The other dimensionless parameter values are determined from the data given in the first column in Table 1. 


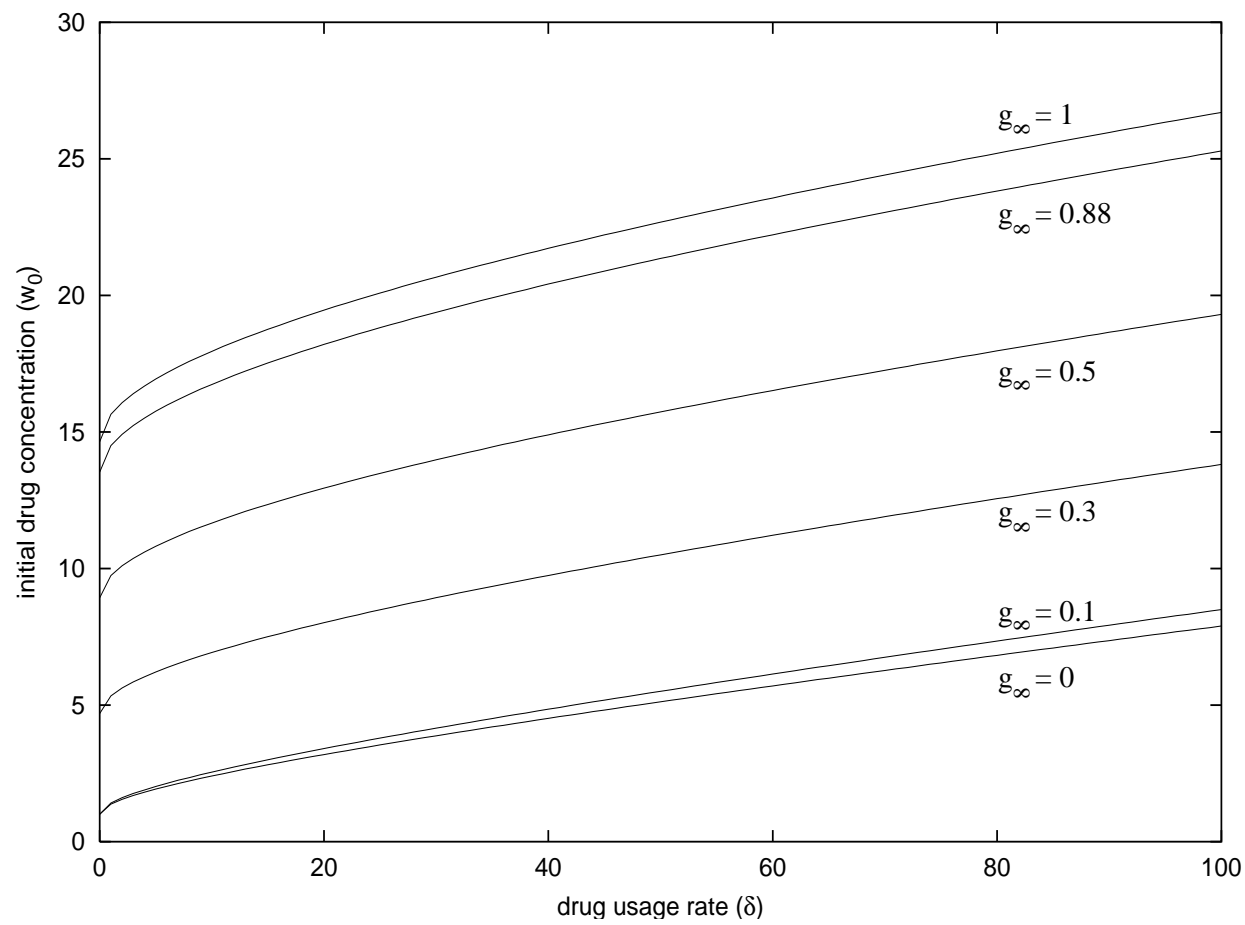

Figure 6: Plot of the bifurcation curves in $\delta-w_{0}$ space for various values of $g_{\infty}$. Parameter regimes above each curve result ultimately result in an exponential decline of the population, below each curve the population will ultimately grow exponentially. The other dimensionless parameter values are determined from the data given in the first column in Table 1. 